\title{
Sterile neutrino dark matter in left-right theories
}

\author{
Jeff A. Dror, ${ }^{a, b}$ David Dunsky, ${ }^{a, b}$ Lawrence J. Hall ${ }^{a, b}$ and Keisuke Harigaya ${ }^{c}$ \\ ${ }^{a}$ Department of Physics, University of California, \\ Berkeley, California 94720, U.S.A. \\ ${ }^{b}$ Theoretical Physics Group, Lawrence Berkeley National Laboratory, \\ Berkeley, California 94720, U.S.A. \\ ${ }^{c}$ School of Natural Sciences, Institute for Advanced Study, \\ Princeton, New Jersey 08540, U.S.A. \\ E-mail: jdror@berkeley.edu, ddunsky@berkeley.edu, ljh@berkeley.edu, \\ keisukeharigaya@ias.edu
}

ABStRACT: $\mathrm{SU}(2)_{L} \times \mathrm{SU}(2)_{R}$ gauge symmetry requires three right-handed neutrinos $\left(N_{i}\right)$, one of which, $N_{1}$, can be sufficiently stable to be dark matter. In the early universe, $W_{R}$ exchange with the Standard Model thermal bath keeps the right-handed neutrinos in thermal equilibrium at high temperatures. $N_{1}$ can make up all of dark matter if they freeze-out while relativistic and are mildly diluted by subsequent decays of a long-lived and heavier right-handed neutrino, $N_{2}$. We systematically study this parameter space, constraining the symmetry breaking scale of $\mathrm{SU}(2)_{R}$ and the mass of $N_{1}$ to a triangle in the $\left(v_{R}, M_{1}\right)$ plane, with $v_{R}=\left(10^{6}-3 \times 10^{12}\right) \mathrm{GeV}$ and $M_{1}=(2 \mathrm{keV}-1 \mathrm{MeV})$. Much of this triangle can be probed by signals of warm dark matter, especially if leptogenesis from $N_{2}$ decay yields the observed baryon asymmetry. The minimal value of $v_{R}$ is increased to $10^{8} \mathrm{GeV}$ for doublet breaking of $\mathrm{SU}(2)_{R}$, and further to $10^{9} \mathrm{GeV}$ if leptogenesis occurs via $N_{2}$ decay, while the upper bound on $M_{1}$ is reduced to $100 \mathrm{keV}$. In addition, there is a component of hot $N_{1}$ dark matter resulting from the late decay of $N_{2} \rightarrow N_{1} \ell^{+} \ell^{-}$that can be probed by future cosmic microwave background observations. Interestingly, the range of $v_{R}$ allows both precision gauge coupling unification and the Higgs Parity understanding of the vanishing of the Standard Model Higgs quartic at scale $v_{R}$. Finally, we study freeze-in production of $N_{1}$ dark matter via the $W_{R}$ interaction, which allows a much wider range of $\left(v_{R}, M_{1}\right)$.

Keywords: Beyond Standard Model, Cosmology of Theories beyond the SM, Neutrino Physics

ArXiv ePrint: 2004.09511 


\section{Contents}

1 Introduction 1

2 Left-right models and neutrino masses 2

$\begin{array}{lll}3 & N_{1} \text { stability } & 5\end{array}$

4 Relativistic freeze-out and dilution $\quad 7$

5 Signals and future probes $\quad 10$

$\begin{array}{lll}5.1 \text { Warmness } & 10\end{array}$

$\begin{array}{lll}5.2 \text { Hotness } & 10\end{array}$

$\begin{array}{lll}5.3 & \text { Early matter dominated era } & 12\end{array}$

$\begin{array}{lll}5.4 \text { Leptogenesis } & 13\end{array}$

6 Predictions on $\boldsymbol{v}_{\boldsymbol{R}}$ from UV physics $\quad 13$

$\begin{array}{lll}\text { 6.1 Small Higgs quartic coupling at high energy scales } & 13\end{array}$

$\begin{array}{ll}6.2 \text { Gauge coupling unification } & 14\end{array}$

$\begin{array}{lll}7 & \text { Freeze-in } & 15\end{array}$

$\begin{array}{lll}8 & \text { Conclusions } & 17\end{array}$

$\begin{array}{lr}\text { A Neutrino mass relations } & 18\end{array}$

B A symmetry for the cosmological stability of $N_{1}$

\section{Introduction}

Left-right (LR) symmetry [1-3] is a possible remnant of grand unification [4-7], can restore space-time parity at high energies, solve the strong CP problem [8-12], and explain the small Standard Model (SM) Higgs quartic coupling at high energy scales [12-15]. In LR theories, the electroweak gauge group, $\mathrm{SU}(2)_{L} \times \mathrm{U}(1)_{Y}$, is extended to $\mathrm{SU}(2)_{L} \times \mathrm{SU}(2)_{R} \times$ $\mathrm{U}(1)_{B-L}$, which is broken at a scale $\left(v_{R}\right)$ above the weak scale $(v), v_{R} \gg v$. LR symmetry predicts right-handed neutrinos, which, if their masses and mixing with the left-handed neutrinos are sufficiently small, can be stable. Since right-handed neutrinos are inert under the SM gauge group, they are candidates to make up the observed dark matter (DM) density of the universe. Right-handed neutrino DM belongs to a class of sterile neutrino DM, and we use "right-handed neutrino" and "sterile neutrino" interchangeably.

How can right-handed neutrino DM be populated in the early universe? For large $v_{R}$ and/or small reheating temperatures of the universe, production of right-handed neutrinos 
through the exchange of heavy gauge bosons, $W_{R}$ and $Z_{R}$, is negligible. Right-handed neutrinos can still be produced by their Yukawa coupling with the SM lepton douplets and Higgs [16]. However, this production mechanism is in tension with the constraints from x-ray searches and structure formation of the universe (see e.g. [17]), unless a significant lepton asymmetry is present [18].

Production of right-handed neutrinos by the exchange of $W_{R}$ and $Z_{R}$ becomes increasingly effective for higher reheating temperatures. The resultant abundance reproduces the observed DM density for an appropriate reheating temperature; above this temperature, right-handed neutrinos are overproduced.

In the limit of high reheating temperatures, right-handed neutrinos are thermalized via $W_{R}$ and $Z_{R}$ exchange. The DM phenomenology of LR theories in the case of high reheat temperatures was first studied in [19], which showed that the lightest right-handed neutrino can make up DM if it decouples while relativistic and has its abundance diluted by decays of heavier right-handed neutrinos into the SM bath through an off-shell $W_{L}$ via sterile-active mixing. The requirement that the heavier neutrino freezes-out while relativistic leads to a constraint on the $W_{R}$ mass, $M_{W_{R}} \gtrsim 10^{4} \mathrm{GeV}$, with no clear upper bound.

In this work we study the parameter space of LR models systematically, mainly for reheat temperatures after inflation above the temperatures needed to thermalize the righthanded neutrinos by $W_{R}$ and $Z_{R}$ exchange. As in [19], right-handed neutrinos decouple relativistically, and the unstable but long-lived states decay to dilute the abundance of the stable state to the observed DM abundance. We extend previous work, finding a bounded parameter space from a combination of constraints including enough dilution, Big-Bang Nucleosynthesis (BBN), warm DM, hot DM, and $\Delta N_{\text {eff. }}$ Upper bounds on the DM neutrino mass and on the $\mathrm{SU}(2)_{R}$ symmetry breaking scale, $v_{R}$, result from a detailed analysis of the neutrino mass matrix, which takes a form constrained by LR symmetry. Furthermore, the mass of the lightest active neutrino is constrained to be $\lesssim 10^{-4} \mathrm{eV}$. We discuss how the resulting parameter space will be probed observationally, especially using $21 \mathrm{~cm}$ cosmology, and also how it is further constrained if decays of the long-lived righthanded neutrino generate the observed baryon asymmetry via leptogenesis. The range of $v_{R}$ predicted by the DM abundance is compared to ranges which lead to precision gauge coupling unification and to the observed value of the Higgs boson mass.

In addition, we study the case of lower reheating scales, finding that freeze-in is also a viable option to produce relic right-handed neutrinos. In this case, the sensitivity to the reheat temperature after inflation leads to a wide open parameter space, with values of $v_{R}$ as large as the Planck scale.

\section{Left-right models and neutrino masses}

In this section we summarize the neutrino sector of left-right theories, emphasizing the role played by the LR symmetry. We begin by considering the effective theory of the SM with 3 additional gauge singlets, $N_{i}$, and then introduce LR symmetry. The leading operators in the SM that give rise to masses for neutrinos are bilinear in lepton fields,

$$
-\mathcal{L}_{\mathrm{SM}+\mathrm{N}, \mathrm{eff}} \supset y_{i j}\left(\ell_{i} N_{j}\right) H_{L}+\frac{y_{i j}^{\prime}}{\Lambda}\left(\ell_{i} \ell_{j}\right) H_{L}^{2}+y_{i j}^{\prime \prime} M_{R}\left(N_{i} N_{j}\right)+\text { h.c. } .
$$


where $\ell_{i} \equiv\left(\nu_{i}, e_{i}\right)$ are the three lepton $\mathrm{SU}(2)_{L}$-doublet fields. This involves three independent dimensionless flavor matrices $\left(y, y^{\prime}, y^{\prime \prime}\right)$ and two mass scales: the SM cutoff scale $\Lambda$ and the right-handed neutrino mass scale, $M_{R}$. Without $N_{i}$, the SM only contains the second of these three operators [20], which is sufficient to adequately describe the observed neutrino masses and mixings, once the SM Higgs field $H_{L}$ acquires its vacuum expection value, $v$. When including $N_{i}$, the second term of (2.1) is often neglected, resulting in light neutrino masses from the seesaw mechanism [21-24] if $M_{R} \gg v$.

In this paper we study the extension of the $\mathrm{SM}$ electroweak gauge group to $\mathrm{SU}(2)_{L} \times$ $\mathrm{SU}(2)_{R} \times \mathrm{U}(1)_{B-L}$. This simplifies the representation structure of the quarks and leptons: $q \equiv(u, d)$ and $\ell \equiv(\nu, e)$ transforming as $(2,1)$ under $\mathrm{SU}(2)_{L} \times \mathrm{SU}(2)_{R}$ and $\bar{q} \equiv(\bar{u}, \bar{d})$ and $\bar{\ell} \equiv(N, \bar{e})$ transforming as $(1,2)$. The presence of the right-handed neutrinos is now required by the gauge symmetry, and this is their natural setting. We impose a discrete symmetry that interchanges $\mathrm{SU}(2)_{L} \leftrightarrow \mathrm{SU}(2)_{R}$; the corresponding transformation on the fermions may include spacetime parity, $\ell \leftrightarrow \bar{\ell}^{\dagger}$, or not, $\ell \leftrightarrow \bar{\ell}$.

We do not specify the full structure of the LR symmetric theory, though any such theory must have the $\mathrm{SU}(2)_{R} \times \mathrm{U}(1)_{B-L}$ gauge symmetry broken to hypercharge at some scale $v_{R} \gg v$. We consider the effective field theory below $v_{R}$, assuming that the only fermions relevant for neutrino masses in the effective theory are $\nu_{i}$ and $N_{i}$, and the leptonnumber violating contribution to their masses is generated by a single type of LR symmetric interaction. In this case, the leading operators for neutrino masses are

$$
-\mathcal{L}_{\mathrm{LR}, \text { eff }} \supset y_{i j}\left(\ell_{i} N_{j}\right) H_{L}+\frac{c y_{i j}^{\prime}}{v_{R}}\left(\ell_{i} \ell_{j}\right) H_{L}^{2}+y_{i j}^{\prime(*)} v_{R}\left(N_{i} N_{j}\right)+\text { h.c. } .
$$

If the LR symmetry includes spacetime parity, $y$ is a Hermitian matrix and the complex conjugation is included in the last term; otherwise $y$ is symmetric and the complex conjugation is omitted. Even though LR symmetry has been spontaneously broken, the $\left(\ell_{i} \ell_{j}\right)$ and $\left(N_{i} N_{j}\right)$ flavor matrices are identical, $y_{i j}^{\prime \prime}=y_{i j}^{\prime}$, reflecting the symmetry structure of the full theory. This will have important consequences for the parameter space in which $N_{1}$ can be DM. Furthermore, comparing with (2.1) we find that $M_{R}=v_{R}$ and $\Lambda=v_{R} / c$, where the constant $c$ is discussed below, and is unity in certain theories.

The effective Lagrangian leads to a $6 \times 6$ neutrino mass matrix,

$$
\left(\begin{array}{ll}
\nu_{i} & N_{i}
\end{array}\right)\left(\begin{array}{cc}
c M_{i j} v^{2} / v_{R}^{2} & y_{i j} v \\
y_{j i} v & M_{i j}^{(*)}
\end{array}\right)\left(\begin{array}{c}
\nu_{j} \\
N_{j}
\end{array}\right),
$$

where $M_{i j}=y_{i j}^{\prime} v_{R}$. Without loss of generality we can work in a basis where $y^{\prime}$ is diagonal such that,

$$
M_{i j}=M_{i} \delta_{i j}
$$

with all $M_{i}$ real. Upon integrating out the three heavy states, we obtain a mass matrix for the three light neutrinos:

$$
m_{i j}=\delta_{i j} c \frac{v^{2}}{v_{R}^{2}} M_{i}-y_{i k} v \frac{1}{M_{k}} y_{j k} v \equiv \delta_{i j} m_{\nu, i}^{(5)}-m_{\nu, i j}^{(s s, N)} .
$$


In this basis, in the limit that $y_{i j}$ is diagonal the lepton flavor mixing arises entirely from the charged lepton mass matrix. Our results apply to any LR theory where neutrino physics below $v_{R}$ is described by (2.2), together with the gauge interactions. Our results may not apply if there are additional states below $v_{R}$ (e.g., neutral fermions with bilinear operators mixing with $\nu$ or $N$ ).

We now consider how the effective theory of (2.2) arises in two simple models. We begin with the conventional LR theory with scalar multiplets $\Delta_{L}, \Delta_{R}$ and $\Phi$ which transform as $(3,1),(1,3)$ and $(2,2)$ under $\mathrm{SU}(2)_{L} \times \mathrm{SU}(2)_{R}$, respectively. This leads to the Lagrangian,

$$
-\mathcal{L}_{\mathrm{LR}} \supset y_{i j}\left(\ell_{i} \bar{\ell}_{j}\right) \Phi+y_{i j}^{\prime}\left(\ell_{i} \ell_{j}\right) \Delta_{L}+y_{i j}^{\prime(*)}\left(\bar{\ell}_{i} \bar{\ell}_{j}\right) \Delta_{R}+\text { h.c. }
$$

With this scalar spectrum, the LR symmetry is broken by $\left\langle\Delta_{R}\right\rangle=v_{R}$, giving the $\left(N_{i} N_{j}\right)$ term of (2.2), and $\Phi$ contains the SM Higgs, $H_{L}$, giving the $\left(\ell_{i} N_{j}\right)$ term of (2.2). Finally, $\Delta_{L}$ acquires a mass of order $v_{R}$ and, when it is integrated out of the theory, leads to the $\left(\ell_{i} \ell_{j}\right)$ term of (2.2) via the quartic interaction $\lambda_{L R} \Delta_{L} \Delta_{R} \Phi^{\dagger} \Phi$. The constant $c$ is proportional to $\lambda_{L R}$, and hence $c$ is typically of order unity or smaller; $c \gg 1$ requires fine-tuning the mass of $\Delta_{L}$ to be far below $v_{R}$ and we do not consider this possibility.

There is a structurally simpler LR model involving just two scalar multiplets $H_{L}$ and $H_{R}$ transforming as $(2,1)$ and $(1,2)$ under $\mathrm{SU}(2)_{L} \times \mathrm{SU}(2)_{R}$. This theory has the virtue that, if the LR symmetry is taken to include spacetime parity, it solves the strong CP problem [10-12]. Furthermore, the vanishing of the SM Higgs quartic coupling at high energies can be understood in this theory from the Higgs Parity mechanism [12]. The pure doublet symmetry breaking leads to leptonic interactions relevant for neutrino masses above $v_{R}$ of the form

$$
-\mathcal{L}_{\mathrm{LR}} \supset f_{i j} \frac{1}{\Lambda}\left(\ell_{i} \bar{\ell}_{j}\right) H_{L} H_{R}+f_{i j}^{\prime} \frac{1}{\Lambda}\left(\ell_{i} \ell_{j}\right) H_{L}^{2}+f_{i j}^{\prime(*)} \frac{1}{\Lambda}\left(\bar{\ell}_{i} \bar{\ell}_{j}\right) H_{R}^{2}+\text { h.c. },
$$

where $\Lambda$ is the UV cutoff for this theory. Inserting the LR symmetry breaking scale, $\left\langle H_{R}\right\rangle=v_{R}$, immediately gives $(2.2)$, with $y_{i j}^{(\prime)}=f_{i j}^{(\prime)} v_{R} / \Lambda$, and the added prediction that $c=1$.

Right-handed neutrino $\mathrm{DM}$ in the $\mathrm{keV}$ to $\mathrm{MeV}$ mass range requires extremely small numbers, whether in the context of $(\mathrm{SM}+N)$ or a LR theory. The requirement that $N_{1}$ is sufficiently light requires (in a LR theory $y^{\prime \prime}=y^{\prime}$ ),

$$
y_{11}^{\prime \prime} \sim\left\{\begin{array}{ll}
10^{-20}\left(\frac{M_{1}}{10 \mathrm{keV}}\right)\left(\frac{10^{15} \mathrm{GeV}}{M_{R}}\right)\left(\mathrm{SM}+N_{i}\right) \\
10^{-15}\left(\frac{M_{1}}{10 \mathrm{keV}}\right)\left(\frac{10^{10} \mathrm{GeV}}{v_{R}}\right)(\mathrm{LR})
\end{array},\right.
$$

where we have normalized $v_{R}$ to a scale intermediate between the weak and grand unification scales, which will follow from an $N_{1}$ DM production mechanism studied below. Right-handed neutrino DM runs counter to the simple seesaw understanding of why the neutrinos are much lighter than the charged fermion masses [21-24]. In (2.1), taking $\Lambda \gg 10^{15} \mathrm{GeV}$ so that the second term is irrelevant, and taking $M_{R} \sim 10^{15} \mathrm{GeV}$, gives the 
observed neutrino masses for $y_{i j}$ and $y_{i j}^{\prime \prime}$ of order unity. Nevertheless, given the exceptionally small numbers that arise in these theories to understand the weak scale $\left(10^{-32}\right)$ and the cosmological constant $\left(10^{-120}\right)$, it seems worth pursuing right-handed neutrino DM, especially in LR theories where their existence is a necessity.

\section{$\begin{array}{lll}3 & N_{1} & \text { stability }\end{array}$}

We define $N_{1}$ as a cosmologically stable right-handed neutrino responsible for the DM density of the universe. Even though there is no symmetry that stabilizes $N_{1}$, it may be sufficiently long-lived to be a DM candidate. The dominant decay of $N_{1}$ is driven by $N_{1}-\nu$ mixing controlled by $y_{1 i}$; hence $y_{1 i} \ll 1$ is needed for $N_{1}$ to be long-lived. ${ }^{1}$

The $N_{1}-\nu$ mixing angle is given by

$$
\sin 2 \theta_{1} \equiv \frac{v}{M_{1}} \sqrt{\Sigma_{i}\left|y_{1 i}\right|^{2}}
$$

where $v \simeq 174 \mathrm{GeV}$. The experimental constraints on $\sin 2 \theta_{1}$ arise from two different processes: for $M_{1}$ below about $3 \mathrm{keV}$, the dominant constraint on the sterile-active mixing angle comes from overproducing $N_{1}$ DM via the Dodelson-Widrow mechanism [16]. For heavier $N_{1}$, the dominant constraint comes from overproducing photons by $N_{1}$ DM decays, most prominently through $N_{1} \rightarrow \nu \gamma[25]$ :

$$
\begin{aligned}
\Gamma_{N_{1} \rightarrow \nu \gamma} & \simeq \frac{9 \alpha}{8192 \pi^{4}} \frac{M_{1}^{5}}{v^{4}} \sin ^{2} 2 \theta_{1}, \\
& \simeq\left(1.5 \times 10^{30} \mathrm{sec}\right)^{-1}\left(\frac{M_{1}}{1 \mathrm{keV}}\right)^{5}\left(\frac{\sin ^{2} 2 \theta_{1}}{5 \times 10^{-9}}\right) .
\end{aligned}
$$

As the decay rate is $\propto M_{1}^{5}$ it grows rapidly with $M_{1}$ and is a powerful constraint on the mixing angle for $M_{1} \gtrsim \mathrm{keV}$. Sufficient stability of $N_{1}$ requires $\Gamma_{N_{1} \rightarrow \nu \gamma} \lesssim 1 \times 10^{-27} s^{-1}$ [25] and hence

$$
\left|y_{1 i}\right| \lesssim 10^{-13}\left(\frac{10 \mathrm{keV}}{M_{1}}\right)^{3 / 2} \quad\left(M_{1} \gtrsim 3 \mathrm{keV}\right) .
$$

The combination of the constraints leads to a limit on the mixing angle [25],

$$
\sin ^{2} 2 \theta_{1} \leq 5 \times 10^{-9} \begin{cases}\left(\frac{M_{1}}{3 \mathrm{keV}}\right)^{-1.8} \times D & \text { (Overproduction) } \\ \left(\frac{M_{1}}{3 \mathrm{keV}}\right)^{-5} & \text { (Decay) } .\end{cases}
$$

$D$ is the dilution factor required to reduce a thermal yield of $N_{1}$ to the correct DM abundance. The higher photometric sensitivities of next generation x-ray and gamma-ray telescopes such as ATHENA [26] and e-ASTROGAM [27] may probe an order of magnitude smaller $\sin ^{2} 2 \theta_{1}$ [28]. For $M_{1}>1 \mathrm{MeV}$, the tree-level decay $N_{1} \rightarrow e^{+} e^{-} \nu$ is open and the resultant constraint on $y_{1 i}$ is similar to (3.3).

\footnotetext{
${ }^{1}$ Note that our numbering of SM neutrinos does not necessarily coincide with the neutrino numbering commonly found in the literature.
} 
The smallness of the Yukawa coupling in (3.3) can be explained in the $\mathrm{SM}+N_{i}$ theory by imposing a discrete $Z_{2}$ symmetry under which $N_{i}$ are odd so that the last operator of (2.1) is forbidden, giving $y_{1 i}=0$ and making $N_{1}$ stable. Furthermore, introducing an inert doublet $H_{L}^{\prime}$, that has no vacuum expectation value and is odd under this parity, allows the first operator of (2.1) to be generated from a 1-loop radiative correction [29]. In the LR framework, a $Z_{2}$ symmetry that sets $y_{1 i}=0$ also forbids charged lepton Yukawa couplings. However, in a LR theory one can alternatively impose a discrete $Z_{4 L} \times Z_{4 R}$ symmetry setting $y_{1 i}=0$, guaranteeing cosmologically stable $N_{1}$, while allowing charged lepton masses. We discuss how the model works in appendix B.

If kinematically allowed, $N_{1}$ can also beta decay via $W_{R}$ exchange to $\ell^{ \pm}+$hadron(s), where $\ell^{ \pm}$is any charged lepton, regardless of how small $y_{1 i}$ is. The inclusive decay rate is,

$$
\begin{aligned}
\Gamma_{N_{1} \rightarrow \ell^{ \pm}+\text {hadrons }} & \simeq \frac{3}{1536 \pi^{3}} \frac{M_{1}^{5}}{v_{R}^{4}} \\
& \simeq\left(1.4 \times 10^{24} \mathrm{sec}\right)^{-1}\left(\frac{M_{1}}{150 \mathrm{MeV}}\right)^{5}\left(\frac{v_{R}}{10^{10} \mathrm{GeV}}\right)^{-4} .
\end{aligned}
$$

For sufficiently small $M_{1}$ or large $v_{R}$, this is below the observational upper bounds of $\sim 10^{25} \mathrm{sec}[30] .{ }^{2}$ Here the decay rate is estimated in the quark picture, but the interpolation to the meson regime $M_{1} \gtrsim m_{\pi}$ is correct at the order of magnitude level.

For $M_{1}$ below the pion mass, beta decay to $\ell^{+} \ell^{-} \nu$ via $W_{R}-W_{L}$ mixing is important. This decay channel is also independent of $y_{1 i}$ and given by

$$
\Gamma_{N_{1} \rightarrow \ell^{+} \ell^{-} \nu} \simeq \frac{\Gamma_{N_{1} \rightarrow \ell^{ \pm}+\text {hadrons }}}{3} \times \begin{cases}1 & :(2,2) \text { Breaking } \\ \left(\frac{1}{16 \pi^{2}} \frac{m_{b} m_{t}}{v^{2}} \ln \left(\frac{\Lambda}{v}\right)\right)^{2} & :(2,1)+(1,2) \text { Breaking. }\end{cases}
$$

When the electroweak symmetry is broken by an $\mathrm{SU}(2)_{L} \times \mathrm{SU}(2)_{R}$ bi-fundamental scalar, its vacuum expectation value gives a $W_{R}-W_{L}$ mixing at tree-level. If the electroweak symmetry is broken by an $\mathrm{SU}(2)_{L}$ doublet scalar, the mixing is generated by a top/bottom quark loop. The quantum correction is logarithmically divergent in the effective theory where the quark masses are given by dimension- 5 operators, similar to eq. (2.7). The scale $\Lambda$ that cuts off the divergence is model-dependent, but is close to $v_{R}$ since the top and bottom Yukawa couplings are not small.

The $W_{R}-W_{L}$ mixing also induces the decay of $N_{1}$ into $\nu \gamma[19,31,32]$. Connecting the $\ell^{+} \ell^{-}$in the beta-decay diagram and attaching an external photon to this loop gives a decay rate

$$
\Gamma_{N_{1} \rightarrow \nu \gamma} \simeq \frac{\alpha}{4 \pi} \frac{m_{\tau}^{2}}{M_{1}^{2}} \Gamma_{N_{1} \rightarrow \ell^{+} \ell^{-}}
$$

which is observationally limited by photon searches to be less than about $10^{-27} \mathrm{~s}^{-1}$.

\footnotetext{
${ }^{2}$ Although $N_{1}$ decays via $W_{R}$ to charged pions which then decay to muons, for $M_{1} \gg m_{\pi}$, there may be a large number of neutral pions in the decay shower, which subsequently decay to hard photons and yield slightly stronger constraints on the $N_{1}$ lifetime [30].
} 


\section{Relativistic freeze-out and dilution}

The right-handed neutrinos couple to the SM bath via $W_{R}$ exchange. If the reheat temperature of the universe after inflation is sufficiently high,

$$
T_{\mathrm{RH}}^{\mathrm{inf}} \gtrsim 10^{8} \mathrm{GeV}\left(\frac{v_{R}}{10^{10} \mathrm{GeV}}\right)^{4 / 3},
$$

the right-handed neutrinos reach thermal equilibrium and subsequently decouple with a thermal yield $Y_{\mathrm{th}} \simeq 0.004 .^{3}$ For $N_{1}$ to have the observed DM abundance requires $m_{N_{1}} \simeq$ $100 \mathrm{eV}$; however, such light sterile neutrino DM is excluded by the Tremaine-Gunn [33-35] and warmness [36-39] bounds; see [25] for a recent review.

Nevertheless, it is still possible to realize $N_{1}$ as DM with $M_{1} \gtrsim \mathrm{keV}$, if they decouple relativistically from the thermal bath and their abundance is diluted. If another right-handed neutrino, $N_{2}$, is sufficiently long-lived such that it comes to dominate the energy density of the universe and produces entropy when it decays, it can dilute the DM abundance and cool $N_{1}$ below warmness bounds [19,40]. The relic density of $N_{1}$ is

$$
\begin{aligned}
\frac{\rho_{N_{1}}}{s} & =1.6 \frac{3}{4} \frac{M_{1}}{M_{2}} T_{\mathrm{RH}}, \\
\Rightarrow \frac{\Omega_{N_{1}}}{\Omega_{\mathrm{DM}}} & \simeq\left(\frac{M_{1}}{10 \mathrm{keV}}\right)\left(\frac{300 \mathrm{GeV}}{M_{2}}\right)\left(\frac{T_{\mathrm{RH}}}{10 \mathrm{MeV}}\right),
\end{aligned}
$$

where the numerical factor 1.6 is taken from [41], $\rho_{N_{1}}$ is the energy density, $s$ is the entropy density, $\Omega_{\mathrm{DM}} \simeq 0.25$ is the observed cosmic relic abundance, and $T_{\mathrm{RH}}$ is the decay temperature of $N_{2}$, as set by its total decay rate $\Gamma_{N_{2}}$

$$
T_{\mathrm{RH}}=\left(\frac{10}{\pi^{2} g_{*}}\right)^{1 / 4} \sqrt{\Gamma_{N_{2}} M_{\mathrm{Pl}}}
$$

These formulae are also applicable to the case where $N_{3}$ first dominates the universe and decays to create entropy, and later $N_{2}$ dominates and creates entropy again. Inserting the warmness bound on $N_{1},\left(M_{1}>2 \mathrm{keV}\right.$, see section 5.1), and the reheating bound from hadronic decays of $N_{2}$ during $\mathrm{BBN}\left(T_{\mathrm{RH}}>4 \mathrm{MeV}\right)[42-44],{ }^{4}$ into (4.2) requires ${ }^{5}$

$$
M_{2} \gtrsim 24 \mathrm{GeV} \text {. (Warmness, reheating, DM abundance). }
$$

\footnotetext{
${ }^{3}$ The analysis is this section is also applicable to lower $T_{\mathrm{RH}}^{\mathrm{inf}}$ as long as $N_{1}$ and $N_{2}$ are frozen-in from $W_{R}$ exchange, and $N_{1}$ is overproduced as DM (see eq. (7.2)). In such a scenario, the required dilution to realize $N_{1} \mathrm{DM}$ is diminished, and hence the warmness constraints on $N_{1}$ slightly increase above $2 \mathrm{keV}$. See figure 4 for the warmness constraints on a pure freeze-in cosmology without any dilution.

${ }^{4}$ Low reheating temperatures can also affect the CMB since some decays occur after neutrinos decouple, reducing the effective number of neutrinos [42, 43, 45]. In our case, $N_{2}$ also decays into neutrinos and the bound from the CMB, $T_{\mathrm{RH}}>4 \mathrm{MeV}[46]$, may be relaxed.

${ }^{5}$ Ref. [47] points out that if the mass eigenstate $N_{1}$ forms an $\mathrm{SU}(2)_{R}$ doublet with the mass eigenstate $\tau$, it might be possible for $N_{1}$ to decouple earlier than $N_{2}$ because of the Boltzmann-suppressed density of $\tau$ relative to $\mu$ or $e$. This reduces the relic density of $N_{1}$ compared to $N_{2}$ which relaxes the necessary dilution from $N_{2}$ by a factor of 3-4 and hence lowers the bound on $M_{2}$ from $24 \mathrm{GeV}$ to $6-8 \mathrm{GeV}$. However, if $N_{2}$ decouples after $N_{1}$, its density is Boltzmann-suppressed since $M_{2} \gg m_{\tau}$, making dilution ineffective. Consequently, we find that the potential relaxation of the bound on $M_{2}$ (4.4) unattainable. Ref. [47] also points out that if $N_{2}$ forms an $\mathrm{SU}(2)_{R}$ doublet with $\mu$ and $M_{2} \simeq m_{\mu}+m_{\pi} \simeq 250 \mathrm{MeV}$, the decay rate of $N_{2}$ via $W_{R}$ exchange is suppressed by a small phase space, allowing $N_{2}$ to be long-lived and provide sufficient dilution even for $v_{R}$ around the TeV scale. Since we find that the possible relaxation of the lower bound on $M_{2}$ does not work, we also cannot confirm this claim.
} 
There are several possible decay modes for $N_{2}$, and which one dominates varies with $M_{2}$. $N_{2}$ can always beta decay through $W_{R}$ exchange into right-handed fermions, $N_{2} \rightarrow$ $\left(\ell^{+} \bar{u} d, \ell^{-} u \bar{d}\right)$ and $N_{2} \rightarrow N_{1} \ell^{+} \ell^{-}$. These decay channels are unavoidable as they are independent of the free-parameter $y_{2 i}$, and prevent $N_{2}$ from efficiently diluting $N_{1}$ in some regions of parameter space. The $N_{2}$ decay rate via $W_{R}$ exchange is

$$
\Gamma_{N_{2} \rightarrow N_{1} \ell^{+} \ell^{-}}+\Gamma_{N_{2} \rightarrow\left(\ell^{+} \bar{u} d, \ell^{-} u \bar{d}\right)}=\frac{1}{1536 \pi^{3}} \frac{M_{2}^{5}}{v_{R}^{4}} \times 20 .
$$

In addition, when $M_{2} \gtrsim v, N_{2}$ can decay at tree-level via $N_{2} \rightarrow \nu h, \nu Z, \ell^{ \pm} W^{\mp}$ while for $M_{2} \lesssim v, N_{2}$ can beta decay through $W_{L} / Z$ exchange and active-sterile mixing to SM fermions, $N_{2} \rightarrow \ell u d, \ell^{+} \ell^{-} \nu, \nu \nu \bar{\nu}$. These decay rates are given by

$$
\begin{array}{rlrl}
\Gamma_{N_{2} \rightarrow \ell H_{L}} & =\frac{1}{8 \pi} \sum_{i}\left|y_{2 i}\right|^{2} M_{2} & & \left(M_{2} \gtrsim v\right) \\
\Gamma_{N_{2} \rightarrow\left(\ell^{+} \bar{u} d, \ell^{+} \ell^{-} \bar{\nu}, \nu \nu \bar{\nu} \text { or h.c. }\right)} & \simeq \frac{171}{8} \frac{1}{1536 \pi^{3}} \frac{M_{2}^{3}}{v^{2}} \sum_{i}\left|y_{2 i}\right|^{2} & \left(M_{2} \lesssim v\right) .
\end{array}
$$

For the latter we add up the results in $[19,48]$ in the limit of a vanishing Weinberg angle, for simplicity. In either case, $y_{2 i}$ must be sufficiently small so that $N_{2}$ dominates the energy density of the universe before decaying. Diluting $N_{1}$ to the observed DM abundance requires

$$
\left|y_{2 i}\right| \lesssim \begin{cases}3 \times 10^{-10}\left(\frac{M_{2}}{24 \mathrm{GeV}}\right)^{-1 / 2}\left(\frac{M_{1}}{2 \mathrm{keV}}\right)^{-1} & \left(M_{2} \lesssim v\right) \\ 1 \times 10^{-11}\left(\frac{M_{2}}{v}\right)^{1 / 2}\left(\frac{M_{1}}{2 \mathrm{keV}}\right)^{-1} & \left(M_{2} \gtrsim v\right) .\end{cases}
$$

The equality sign applies when the contribution to the $N_{2}$ decay rate from $W_{R}$ exchange, (4.5), is sub-dominant.

In appendix A, we use the above results, together with the radiative stability bound on $N_{1}$, to derive constrains on the neutrino mass matrix of $(2.5)$.

1. For $M_{1}<M_{3}$, we show that the lightest neutrino mass eigenstate is closely aligned with $\nu_{1}$ and has a mass $m_{1} \ll \sqrt{\Delta m_{\text {sol }}^{2}}$. In the case that $M_{3}>M_{2}$, the other two mass eigenstates are very close to $\nu_{2}$ and $\nu_{3}$ and have masses $m_{2}=c\left(v^{2} / v_{R}^{2}\right) M_{2}$ and $m_{3}=c\left(v^{2} / v_{R}^{2}\right) M_{3}-y_{33}^{2} v^{2} / M_{3}$. For $M_{3}<M_{2}$, the $(2,3)$ entry of the mass matrix may be non-negligible, so that the two heavy active mass eigenstates are each linear combinations of $\nu_{2,3}$. In this case, we are able to derive a relation between the scale of their masses and $M_{2}: M_{2} \simeq \mu\left(v_{R} / v\right)^{2} c^{-1}$, where $0.01 \mathrm{eV} \lesssim \mu \lesssim 0.10 \mathrm{eV}$. In the rest of the paper we take

$$
M_{2} \simeq m_{2}\left(\frac{v_{R}}{v}\right)^{2} \frac{1}{c}
$$

but, in the case that $M_{3}<M_{2}, m_{2}$ should be taken in the range $(0.01-0.10) \mathrm{eV}$ and not set to an active neutrino mass eigenvalue. 

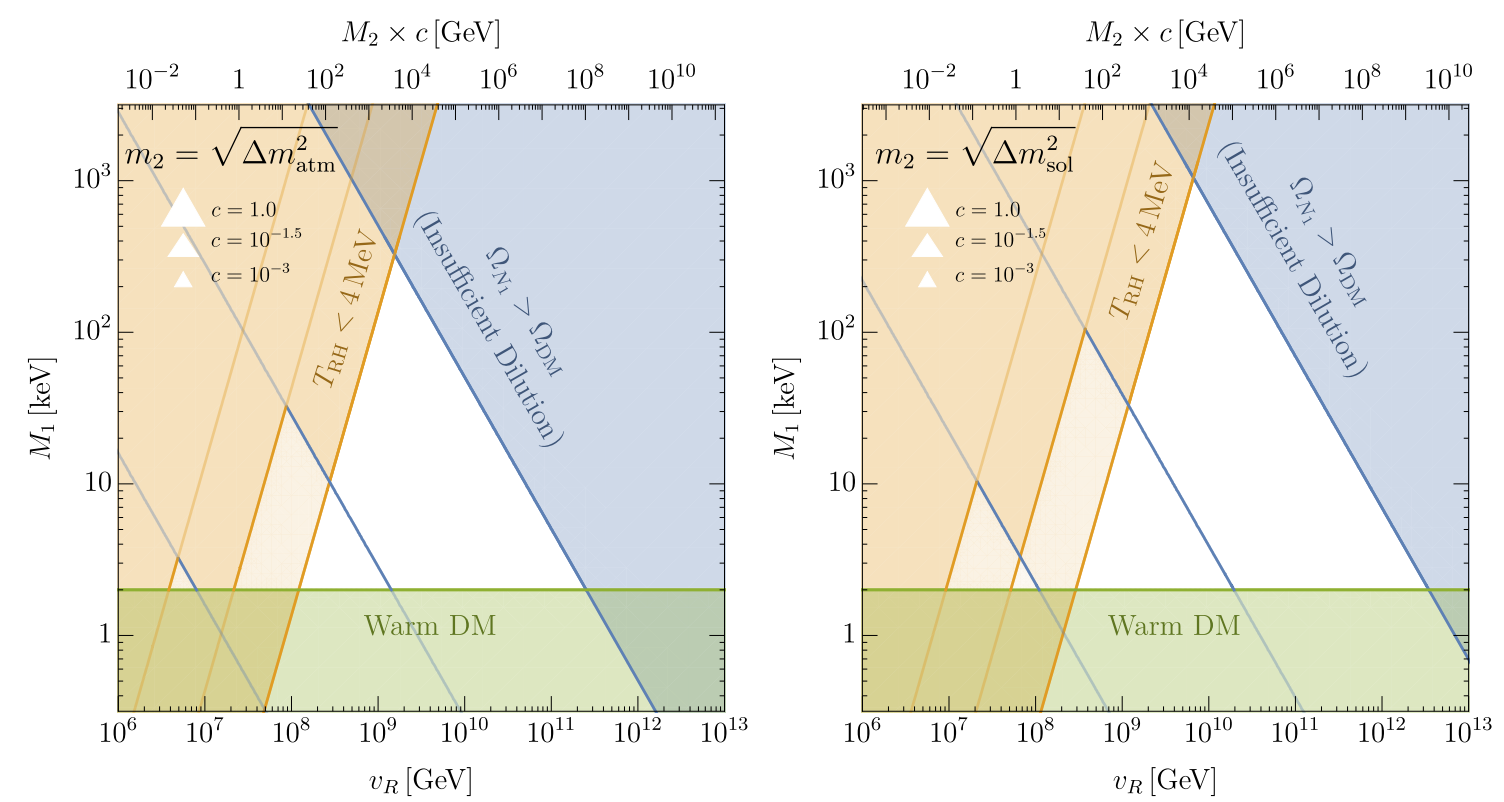

Figure 1. The parameter space of $N_{1}$ DM produced by relativistic freeze-out and dilution from $N_{2}$ decay: constraints on the LR symmetry breaking scale $v_{R}$ and the mass $N_{1}$. The constraints from warm DM are in green, Big Bang Nucleosynthesis in orange, and insufficient dilution in blue. The constraints depend on the LR-model dependent parameter $c \lesssim 1$. Left: we fix the $\nu_{2}$ mass by the atmospheric neutrino mass difference, $m_{2}=\sqrt{\Delta m_{\mathrm{atm}}^{2}}$. Right: we fix the $\nu_{2}$ mass by the solar neutrino mass difference, $m_{2}=\sqrt{\Delta m_{\mathrm{sol}}^{2}}$.

2. For $M_{1}>M_{3}$, we show that the lightest neutrino mass is much smaller than $\sqrt{\Delta m_{\text {sol }}^{2}}$ and that $M_{2}$ is given by eq. (4.9), with the parameter $c$ replaced by a parameter $c_{\text {eff }}<c$.

In figure 1 , we show the constraints on $\left(v_{R}, M_{1}\right)$ when $m_{2}=\sqrt{\Delta m_{\text {atm }}^{2}}$ (left) and $m_{2}=\sqrt{\Delta m_{\mathrm{sol}}^{2}}$ (right). In the orange shaded region, the required $T_{\mathrm{RH}}$ is below $4 \mathrm{MeV}$, which is excluded by hadronic decays of $N_{2}$ during BBN [42, 43]. The green-shaded region is excluded due to the warmness of $N_{1}$ affecting large scale structure. To the right of the blue line, the beta decay rate of $N_{2}$ via $W_{R}$ exchange is the dominant contribution to $\Gamma_{N_{2}}$; here, the dilution of $N_{1}$ is chiefly through $N_{2} \rightarrow N_{1} \ell^{+} \ell^{-}$and $N_{2} \rightarrow\left(\ell^{+} \bar{u} d, \ell^{-} u \bar{d}\right)$. Using (4.9), these decay rates scale as a positive power of $M_{2}$ and hence $v_{R}$. Within the blue-shaded region, the $N_{2}$ decay rate becomes too fast to efficiently dilute the $N_{1}$ energy density.

The blue line itself is an interesting region of parameter space, which does not require any tuning but simply corresponds to the limit where the the dominant decay is set entirely by the $W_{R}$ exchange terms in (4.5). In this limit the $N_{1}$ abundance has two contributions: from $N_{2}$ decay through $N_{2} \rightarrow N_{1} \ell^{+} \ell^{-}$as well as the abundance from relativistic decoupling. While the latter is the dominant component, the former can also make up a significant component of DM, which can be probed by future experiments as discussed in section 5.2.

As can be seen from figure 1, the allowed region of frozen-out $N_{1}$ DM from LR theories forms a bounded triangle in the $v_{R}-M_{1}$ plane. The position and size of the triangle depends 
on $c$, such that the allowed region shrinks in size and shifts to lower $v_{R}$ for smaller $c$. This is because the $\Omega_{N_{1}}>\Omega_{\mathrm{DM}}$ bound depends more sensitively on $M_{2}$ (and hence $c$ ) than the $T_{\mathrm{RH}}<4 \mathrm{MeV}$ bound. We show the effect of $c$ on the allowed region for three values of $c$ : one near the experimental minimum, one near the natural maximum, and one in between. As can be seen by the smallest triangle of figure 1, the allowed region of $N_{1} \mathrm{DM}$ disappears for $c \lesssim 1 \times 10^{-4}$, placing an experimental lower bound on $v_{R} \gtrsim 10^{6} \mathrm{GeV}$. Similarly, the naturalness argument discussed in section 2 limits $c$ near unity, and an upper bound on $v_{R} \lesssim 10^{13} \mathrm{GeV}$ as shown by the largest triangle of figure 1 . For the remainder of this paper, we conservatively focus on the case $c=1$, the largest naturally allowed parameter space of $N_{1}$ DM, when considering signals and future experimental probes.

\section{Signals and future probes}

So far we have focused on the current constraints on sterile neutrino DM in general LR theories and found freeze-out to be a viable option as long as the $c$ parameter, characterizing the seesaw contribution to the light neutrino masses, is not too small. In this section, we discuss how future observations can probe the parameter space through dark radiation, warm DM, and additional structure on very small scales. In addition, the requirement of viability of leptogenesis greatly restricts the parameter space.

\subsection{Warmness}

The free-streaming length of thermally produced $N_{1}$ can be large if $N_{1}$ is light. When $M_{1}$ is $\mathcal{O}(\mathrm{keV})$, the free-streaming length of $N_{1}$ approaches the size of galactic mass perturbations, suppressing the matter power spectrum on scales $k \gtrsim 0.1 \mathrm{Mpc}^{-1}$ [49-53]. A suppression can be observed through large scale structure surveys, perturbations in the cosmic microwave background (CMB), or absorption of low-redshift Lyman- $\alpha$ photons by neutral hydrogen (a tracer of DM) in the intergalactic medium [54-57]. For $N_{1}$ that was thermally produced and diluted to the observed DM abundance, the bounds are at $\mathcal{O}(1-5 \mathrm{keV})$ range. We adopt $M_{1} \gtrsim 2 \mathrm{keV}$ as our constraint in the dark green region of figure 2. Future 21$\mathrm{cm}$ cosmology experiments, which can trace early star and galaxy formation at cosmic dawn, are anticipated to probe the matter power spectrum on scales $k \gtrsim 50 \mathrm{Mpc}^{-1}$. If no suppression on such scales is observed, searches would constrain $M_{1} \gtrsim 14 \mathrm{keV}$ [58], which we show with the dashed green region of figure 2 .

\subsection{Hotness}

Although $N_{1} \mathrm{DM}$ is dominantly produced thermally, a subdominant fraction is always produced non-thermally (see section 4). Specifically, the beta decay $N_{2} \rightarrow N_{1} \ell^{+} \ell^{-}$produces relativistic $N_{1}$. This non-thermal population of $N_{1}$ becomes non-relativistic at temperatures $\mathcal{O}(\mathrm{eV})$ and contributes a hot component of DM. Constraints on hot DM are conventionally given in terms of the effective number of neutrino species, $\Delta N_{\text {eff }}$, which parameterizes its energy density while relativistic, and the effective neutrino mass, $m_{\nu \text {,eff }}$, which parameterizes its energy density when it has become non-relativistic matter $[56,59]$. 

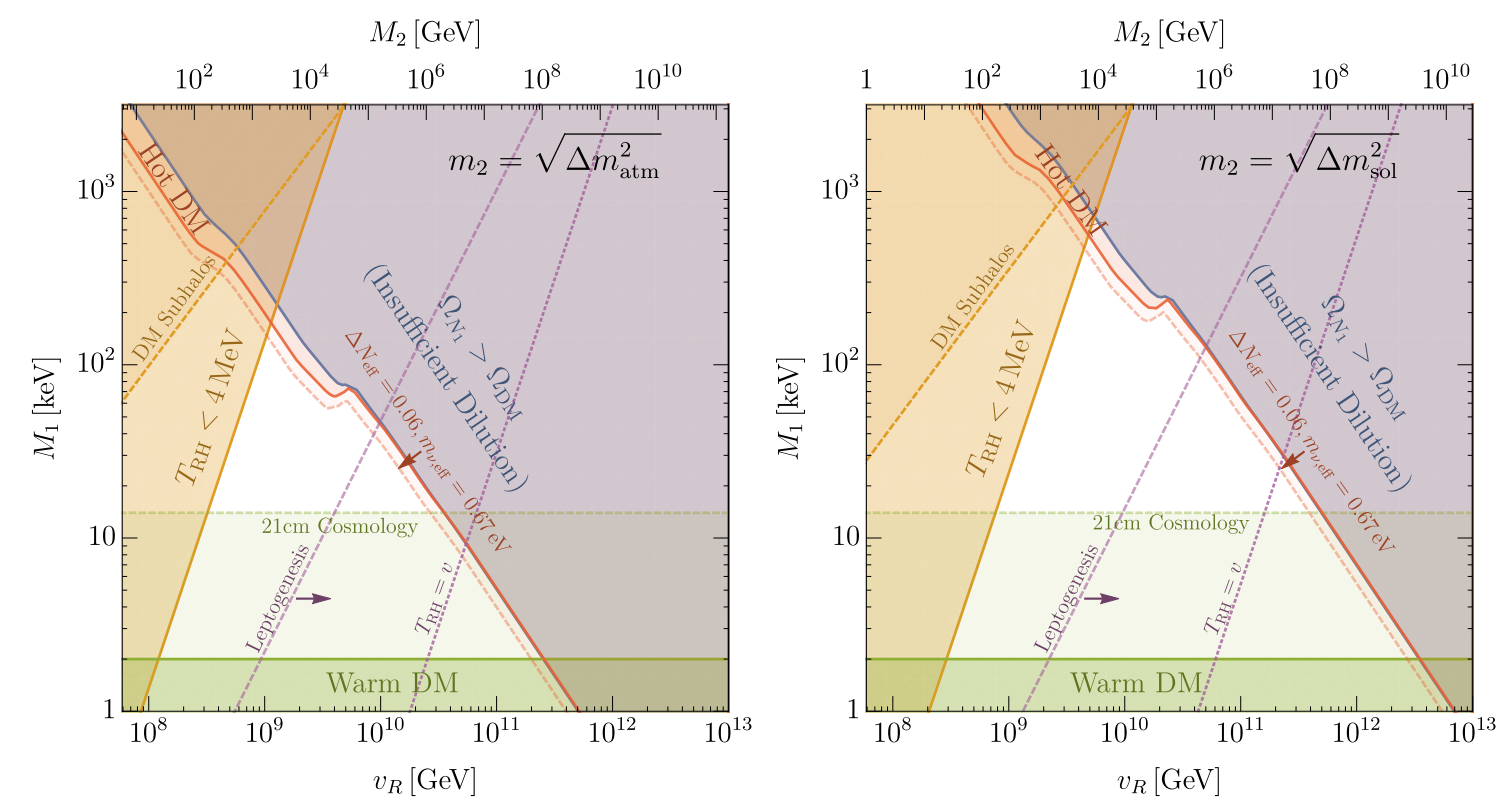

Figure 2. The parameter space of $N_{1}$ DM produced by relativistic freeze-out and dilution from $N_{2}$ decay in terms of the left-right symmetry breaking scale, $v_{R}$, and the mass of $N_{1}, M_{1}$, for $c=1$. We show constraints from $N_{2}$ decaying after Big Bang Nucleosynthesis (orange), decaying too early to provide sufficient $N_{1}$ dilution (blue), warm DM bounds (green), and hot DM bounds (red). In addition we show prospects of future surveys of $T_{\mathrm{RH}}$ from pulsar timing on DM subhalos (dashed orange), improved searches for hot DM from CMB telescopes (dashed red), and warm DM from 21-cm cosmology (dashed green). Lastly, to the left of the dashed purple curve labeled 'Leptogenesis', the baryon asymmetry produced by $N_{2}$ decays is insufficient due to dilution and sphalerons, even with $\epsilon=1$. Left: we fix the $\nu_{2}$ mass with the atmospheric neutrino mass difference, $m_{2}=\sqrt{\Delta m_{\mathrm{atm}}^{2}}$. Right: we fix the $\nu_{2}$ mass with the solar neutrino mass difference, $m_{2}=\sqrt{\Delta m_{\mathrm{sol}}^{2}}$.

For LR models, this is given by

$$
\begin{aligned}
\Delta N_{\mathrm{eff}} & =\frac{1}{3} \operatorname{Br}\left(N_{2} \rightarrow N_{1} \ell^{+} \ell^{-}\right)\left(\frac{g_{*, \mathrm{eq}}^{4}}{g_{*, \mathrm{~T}_{\mathrm{RH}}}}\right)^{\frac{1}{3}} \frac{4}{7}\left(\frac{4}{11}\right)^{-\frac{4}{3}} \\
& \simeq 0.97 \operatorname{Br}\left(N_{2} \rightarrow N_{1} \ell^{+} \ell^{-}\right)\left(\frac{106.75}{g_{*, \mathrm{~T}_{\mathrm{RH}}}}\right)^{\frac{1}{3}}, \\
m_{\nu, \mathrm{eff}} & \equiv 94.1 \mathrm{eV} \Omega_{N_{1}, \mathrm{hot}} h^{2} \simeq 11 \mathrm{eV} \mathrm{Br}\left(N_{2} \rightarrow N_{1} \ell^{+} \ell^{-}\right) .
\end{aligned}
$$

When the $W_{R^{-}}$exchange decay is subdominant, $\operatorname{Br}\left(N_{2} \rightarrow N_{1} \ell^{+} \ell^{-}\right)$scales as $M_{1}^{2} v_{R}^{2}$, and it saturates at 0.1 (since $90 \%$ of beta decays produce quarks and no $N_{1}$ ) along the blue curve. Along this line a significant amount of hot DM is predicted: $\Delta N_{\text {eff }} \simeq 0.1$ and $m_{\nu, \text { eff }} \simeq$ $1.1 \mathrm{eV}$. Coincidentally, current limits on the two-dimensional marginalized distribution of $\Delta N_{\text {eff }}$ and $m_{\nu, \text { eff }}$ already require $\Delta N_{\text {eff }} \lesssim 0.1$ and $m_{\nu, \text { eff }} \lesssim 1.0 \mathrm{eV}$ [59], which we indicate by the red-shaded region labeled 'Hot DM' in figure 2 .

CMB Stage IV [60], a collection of future ground based telescopes, will be able to search for hot DM signals inside the currently allowed region. Assuming a null detection, the experiment will be able limit $\Delta N_{\text {eff }} \lesssim 0.06$ [61], which we show by the dashed red region 
of figure 2. ${ }^{6}$ Note that for $v_{R} \lesssim 10^{10} \mathrm{GeV}, T_{\mathrm{RH}}$ occurs below the QCD phase transition, which is accompanied by a sharp decrease of $g_{*, T_{\mathrm{RH}}}$, leading to an enhancement in $\Delta N_{\text {eff }}$ and strengthening the red-shaded region. The limit where the the dominant decay of $N_{2}$ is set by the $W_{R}$ exchange can be probed by CMB Stage IV.

\subsection{Early matter dominated era}

The current bound on the reheat temperature, $T_{\mathrm{RH}} \gtrsim 4 \mathrm{MeV}$, comes from $N_{2}$ decaying during $\mathrm{BBN}$, leading to its decay products altering the neutron to proton ratio enough to conflict with the observed light element abundances [42, 43]. Presently, ideas to probe higher reheat temperatures rely on the cosmological effects of the early matter dominated era, namely the formation of ultra compact DM halos [64, 65]. For example, halos with masses as low as $M_{\text {halo }} \simeq 10^{-10} M_{\odot}$ can be observed with pulsar timing arrays once the Square Kilometer Area [66] is built [67] (in principle, gravitational microlensing could also be used to look for sub-halos from early matter domination, but such halos would typically have concentration parameters of $\mathcal{O}\left(10^{3}\right)$ and would be too diffuse to have a sizable signature [67-69]). The largest DM halo masses are correlated with $T_{\mathrm{RH}}$ since the density perturbations, $k(a) \equiv a H(a)$, which enter the horizon during the early matter dominated era and source the halos, are largest just before reheating:

$$
M_{\text {halo }} \approx \frac{4}{3} \pi k_{\mathrm{RH}}^{-3} \rho_{m, 0} \approx 10^{-10} M_{\odot}\left(\frac{T_{2}}{500 \mathrm{MeV}}\right)^{-3}\left(\frac{g_{* s}\left(T_{2}\right)}{68}\right)\left(\frac{g_{*}\left(T_{2}\right)}{68}\right)^{-3 / 2} .
$$

Here, $k_{\mathrm{RH}}=a\left(T_{\mathrm{RH}}\right) H\left(T_{\mathrm{RH}}\right)$ is the scale of density perturbations entering the horizon at $T_{\mathrm{RH}}$, and $\rho_{m, 0}$ is the present-day mass density of non-relativistic matter [64]. From (5.2), we see that pulsar timing arrays can probe reheat temperatures as high as $\sim 500 \mathrm{MeV}$.

An important caveat to these experimental searches arises when DM has such a large free-streaming length that ultra compact halos cannot form during the early matterdominated era. The free-streaming length of $N_{1}$ DM is [70]

$$
\lambda_{\mathrm{FS}} \equiv \int_{0}^{t_{\mathrm{eq}}} \frac{v(a)}{a} d t \leqq \int_{t_{\mathrm{RH}}}^{t_{\mathrm{eq}}} \frac{v(a)}{a} d t=\frac{1}{H_{\mathrm{RH}} a_{\mathrm{RH}}^{2}} \frac{\left\langle p_{\mathrm{dec}}\right\rangle a_{\mathrm{dec}}}{M_{1}} \ln \left(\frac{h\left(a_{\mathrm{eq}}\right)}{h\left(a_{\mathrm{RH}}\right)}\right)
$$

where $h(a) \equiv \sqrt{a^{2}+\left(\left\langle p_{\operatorname{dec}}\right\rangle a_{\operatorname{dec}} / M_{1}\right)^{2}}+a$ and,

$$
\left\langle p_{\mathrm{dec}}\right\rangle \simeq 3.2 T_{\mathrm{eq}} \frac{a_{\mathrm{eq}}}{a_{\mathrm{dec}}}\left(\frac{g_{* s, \mathrm{eq}}}{g_{* s, \mathrm{dec}}} \frac{\rho_{\mathrm{DM}} / s}{M_{1} Y_{\mathrm{therm}}}\right)^{1 / 3}
$$

is the average momentum of $N_{1}$ upon decoupling from the SM bath. When $\lambda_{\mathrm{FS}} \gtrsim k_{\mathrm{RH}}^{-1}$, gravitational lensing and pulsar timing array searches cannot put a bound on $T_{\mathrm{RH}}$ since ultra compact halo objects do not exist in the present universe [64], as shown by the dashed orange line of figure 2. From this bound, we see that probing reheat temperatures above $4 \mathrm{MeV}$ through observations of ultra compact $\mathrm{DM}$ halos requires $M_{1} \gtrsim \mathrm{MeV}$, which is already excluded by the insufficient dilution of $N_{1}$ DM.

\footnotetext{
${ }^{6}$ Future space based telescopes such as CORE can theoretically detect $m_{\nu \text {,eff }} \sim 0.04 \mathrm{eV}$ at $1 \sigma$, but only if $\Delta N_{\text {eff }} \gtrsim 0.05[62,63]$.
} 


\subsection{Leptogenesis}

Besides providing an excellent DM candidate in the form of $N_{1}$, right-handed neutrinos are also appealing in that they can generate the observed baryon asymmetry via leptogenesis [71]. In a forthcoming paper [72], we show that the decay of a heavier, long-lived right-handed neutrino, $N_{2}$, can not only provide the dilution necessary to realize $N_{1}$ DM, but also generate a large lepton asymmetry. In the usual way, this lepton asymmetry is converted to a baryon asymmetry via electroweak sphalerons, generating the observed baryon asymmetry of our universe. Since the sphaleron process ceases operation at temperatures below the weak scale, baryogenesis is suppressed when $T_{\mathrm{RH}}<v$. In this case, the baryon asymmetry is generated by the fraction $\left(T_{\mathrm{RH}} / v\right)^{2}$ of $N_{2}$ that decay in the $N_{2}$ MD-era before the temperature of the universe falls below the weak scale. ${ }^{7}$ Consequently, the generated baryon asymmetry is

$$
Y_{B}=\frac{28}{79} \times \epsilon \frac{3}{4} \frac{T_{\mathrm{RH}}}{M_{2}}\left(\frac{T_{\mathrm{RH}}}{v}\right)^{2}
$$

where $\epsilon$ is the lepton asymmetry generated per $N_{2}$ decay, and the factor of $28 / 79$ accounts for the conversion of the lepton asymmetry into the baryon asymmetry via sphalerons [74].

Independent of the model, $\epsilon$ is at most unity. ${ }^{8}$ Conservatively taking this maximum $\epsilon$, we see from eq. (5.5) that generating the observed baryon asymmetry, $Y_{B} \simeq 8 \times 10^{-11}$, is impossible when $T_{\mathrm{RH}} \ll v$, as shown by the dashed purple contour of figure 2 . This constraint demonstrates that incorporating leptogenesis into $N_{1}$ DM from LR models severely diminishes the viable parameter space, and that future 21-cm cosmological probes of warm DM can significantly probe this reduced parameter space.

\section{Predictions on $v_{R}$ from UV physics}

The cosmologically allowed region of initially thermalized $N_{1}$ DM in LR theories constrains the $\mathrm{SU}(2)_{R}$ symmetry breaking scale $v_{R}$ well above the electroweak scale. As discussed in section 4 , the viable region of the right-handed breaking scale is $10^{6} \lesssim v_{R} / \mathrm{GeV} \lesssim 10^{13}$, for any $c \leq 1$, and $10^{8} \lesssim v_{R} / \mathrm{GeV} \lesssim 10^{13}$ for the case of $c=1$. In this section, we consider the implications such a breaking scale has on prospective theories behind LR models.

\subsection{Small Higgs quartic coupling at high energy scales}

Intriguingly, this range of $v_{R}$ is predicted independently within 'Higgs-Parity' theories [12-15], a subset of LR models with Higgs-doublets $H_{L}$ and $H_{R}$ and with the LR symmetry spontaneously broken by $\left\langle H_{R}\right\rangle \gg\left\langle H_{L}\right\rangle$. In Higgs-Parity models, the SM Higgs quartic coupling $\lambda$ is predicted to vanish at the scale $v_{R}$. The SM renormalization group

\footnotetext{
${ }^{7}$ When $v \simeq T_{\mathrm{RH}}$, the thermal bath is not primordial but generated by $N_{2}$ itself (see e.g. [70, 73]), and the suppression is $\left(T_{\mathrm{RH}} / v\right)^{4}$.

${ }^{8}$ Large $\epsilon$ requires a large Yukawa coupling $y_{33}$, which naively produces too large SM neutrino masses by the see-saw from $N_{3}$. This can be avoided by a certain structure in $y_{i j}^{\prime}$ and $y_{i j}$. The magnitude of $\epsilon$ is also restricted by the stability of $N_{1}$ against quantum correction from $y_{33}$, further constraining the parameter space. We study this in detail in a future work.
} 


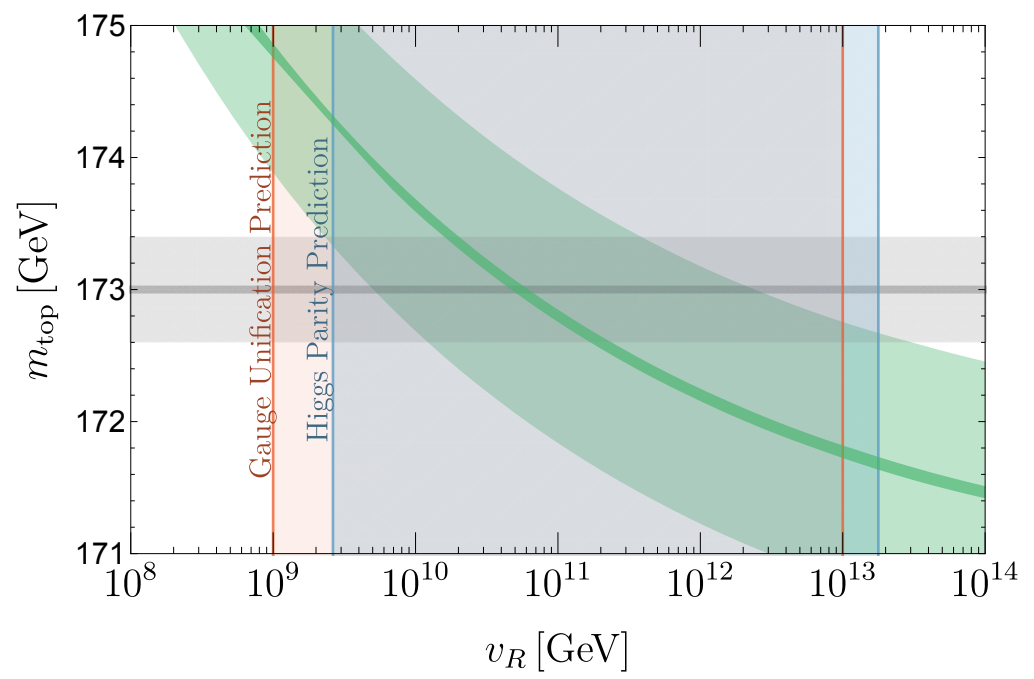

Figure 3. The predicted top quark mass in Higgs Parity theories is shown in green, as a function of the right-handed symmetry breaking scale. The experimentally preferred top mass is shown as a gray band, leading to the preferred range of $v_{R}$ shown by the vertical blue band. The red band shows the range of $v_{R}$ preferred by gauge coupling unification.

flow of $\lambda$ shows that $\lambda=0$ for $10^{9} \lesssim v_{R} / \mathrm{GeV} \lesssim 10^{13}$, with an uncertainty dominantly arising from an uncertainty in the top quark mass [75].

The is shown explicitly in figure 3. The green band shows the relation between $v_{R}$ and the top quark mass, $m_{\text {top }} \cdot{ }^{9}$ The width of this green band arises from the uncertainty of the Higgs mass $m_{h}=125.18 \pm 0.16 \mathrm{GeV}$ and the strong coupling constant $\alpha_{s}\left(m_{Z}\right)=$ $0.1181 \pm 0.0011$ at $2 \sigma[76]$. The preferred value of the top quark mass $(2 \sigma)$ is shown by a horizontal gray band. As a result, the LR symmetry breaking scale $v_{R}$ is predicted to be $10^{9} \lesssim v_{R} / \mathrm{GeV} \lesssim 10^{13}$. The narrower green band shows the relation assuming that the uncertainties shrink to $m_{h}=125.18 \pm .020 \mathrm{GeV}$ and $\alpha_{s}\left(m_{Z}\right)=0.1181 \pm 0.0001$, which is possible through improved lattice calculations, measurements at future lepton colliders, and measurements at HL-LHC [77-79]. The top quark mass can be measured with an accuracy of a few tens of $\mathrm{MeV}$ by $e^{+} e^{-}$colliders [80-83] such as ILC [84], narrowing down the prediction on $v_{R}$ within a few tens of percent, as shown by the narrower gray band. In future work, we will incorporate leptogenesis from $N_{2}$ decays with $N_{1}$ DM within the Higgs Parity framework [72].

\subsection{Gauge coupling unification}

The cosmologically allowed range of $v_{R}$ is also consistent with gauge coupling unification. The LR symmetric gauge group, $\mathrm{SU}(3)_{c} \times \mathrm{SU}(2)_{L} \times \mathrm{SU}(2)_{R} \times \mathrm{U}(1)_{B-L}$ is a subgroup of an $\mathrm{SO}(10)$ unified gauge group. Assuming the minimal symmetry breaking chain containing

\footnotetext{
${ }^{9}$ We ignore a UV completion-dependent part of the threshold correction to $\lambda\left(v_{R}\right)$ from $m_{\text {top }}$ that in some extreme cases can lower the value of $v_{R}$ by $1-2$ orders of magnitude [14].
} 
the LR symmetric gauge group as an intermediate scale gauge group,

$$
\mathrm{SO}(10) \longrightarrow \mathrm{SU}(3)_{c} \times \mathrm{SU}(2)_{L} \times \mathrm{SU}(2)_{R} \times \mathrm{U}(1)_{B-L} \stackrel{v_{R}}{\longrightarrow} \mathrm{SU}(3)_{c} \times \mathrm{SU}(2)_{L} \times \mathrm{U}(1)_{Y}
$$

the scale $v_{R}$ is predicted to be $10^{9} \lesssim v_{R} / \mathrm{GeV} \lesssim 10^{13}[14,85,86]$.

We note, however, that a stable right-handed neutrino, $N_{1}$, is in tension with matter unification. In fact, if the SM quarks and leptons as well as the right-handed neutrinos are unified into a $\mathbf{1 6}$ representation of $\mathrm{SO}(10)$, Yukawa unification naively predicts that the right-handed neutrinos are all heavy and unstable. To evade this naive expectation would require a more sophisticated model in a four-dimensional $\mathrm{SO}(10)$ unified theory. This could be possible with $\mathrm{SO}(10)$ unification in higher dimensions with orbifolding [87-90], where Yukawa couplings do not necessarily unify if matter is localized on gauge symmetry breaking branes [91]. Even if matter lives in the bulk, the SM quarks and leptons as well as the right-handed neutrinos may arise from zero-modes of different 16's by the orbifold projections, as is realized in $\mathrm{SU}(5)[90,91]$ or $\mathrm{SO}(10)$ [92] unification without intermediate gauge symmetry. Breaking of $\mathrm{SO}(10)$ down into LR symmetry by orbifolding is discussed in [93].

\section{$7 \quad$ Freeze-in}

When the reheat temperature of the universe is below the thermalization temperature of the right-handed neutrinos (see (4.1)), neither $N_{1}$ nor $N_{2}$ have a thermal abundance. Instead, the $N_{1}$ abundance is determined by scattering via heavy $W_{R}$ and $Z_{R}$ exchange which, being UV-dominated, depends on the reheating temperature,

$$
\begin{aligned}
& \frac{\rho_{N_{1}}}{s} \simeq 1 \times 10^{-5} \frac{M_{1}\left(T_{\mathrm{RH}}^{\mathrm{inf}}\right)^{3} M_{\mathrm{pl}}}{v_{R}^{4}}, \\
& \Rightarrow \frac{\Omega}{\Omega_{\mathrm{DM}}} \simeq\left(\frac{M_{1}}{150 \mathrm{keV}}\right)\left(\frac{10^{10} \mathrm{GeV}}{v_{R}}\right)^{4}\left(\frac{T_{\mathrm{RH}}^{\mathrm{inf}}}{10^{7} \mathrm{GeV}}\right)^{3} .
\end{aligned}
$$

Freeze-in production from other sources, such as $\ell H \rightarrow N_{1}$, are subdominant since $y_{1 i} \ll 1$ is needed to ensure $N_{1}$ is long-lived. Contributions to the $N_{1}$ abundance may also arise from beta decays of $N_{2}$ and $N_{3}$. These, however, are always subdominant to the direct freeze-in production of $N_{1}$, whether $N_{2,3}$ are produced by the $W_{R}$ interaction or the $\ell N H$ interaction.

In figure 4 , we show the contours of the required reheat temperature after inflation to freeze-in $N_{1} \mathrm{DM}$ for a given $\left(v_{R}, M_{1}\right)$. In the green region, the warmness of $N_{1}$ affects large scale structure. Since frozen-in $N_{1}$ is never diluted, it is warmer than frozen-out $N_{1}$ for a fixed $M_{1}$. More concretely, its free-streaming length is larger by a factor of approximately $\frac{4}{3.2}\left(\frac{M_{1} Y_{\text {therm }}}{\rho_{\mathrm{DM}} / s}\right)^{1 / 3}$, which gives a commensurately stronger warm DM bound compared to figure 2. Here, the factor of $4 / 3.2$ comes from the difference in $\langle p / T\rangle$ for the non-thermal frozen-in distribution, to the thermal frozen-out distribution, as discussed in [94]. In the blue and pink regions, the decay of $N_{1}$ mediated by $W_{R},(3.5)$, or $W_{R}-W_{L}$ 


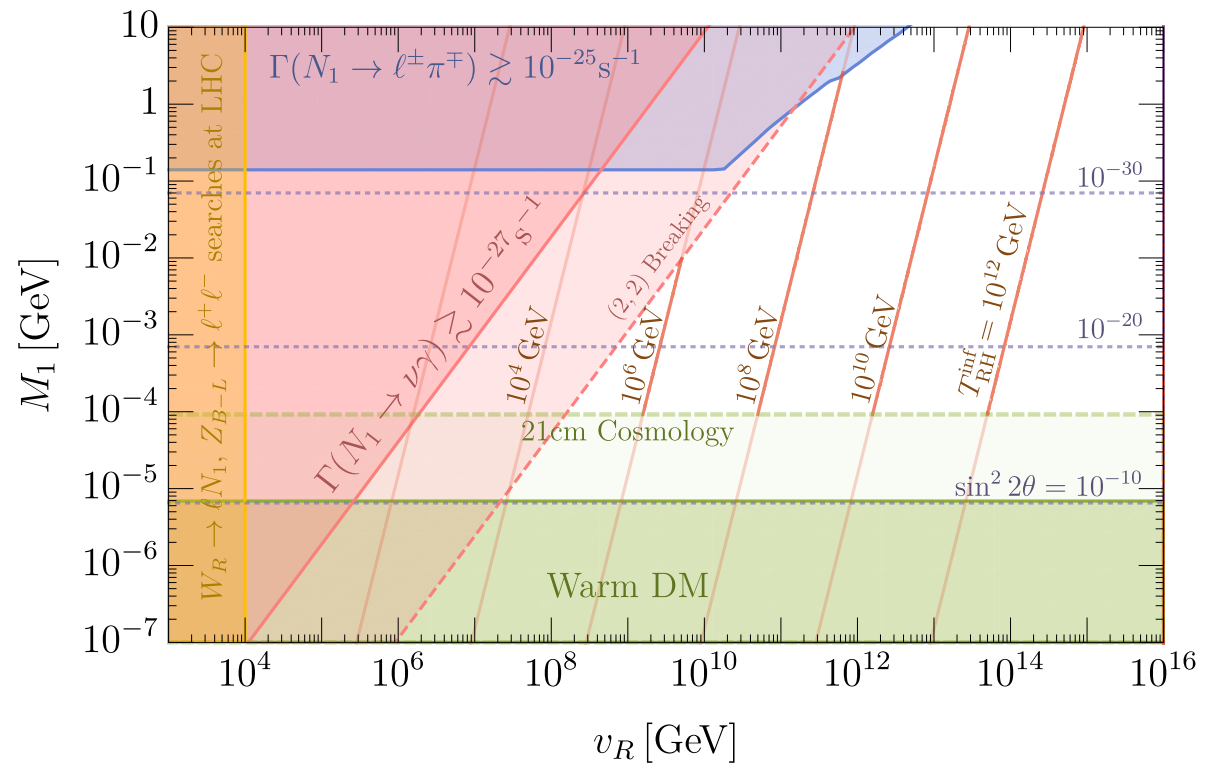

Figure 4. The parameter space for $N_{1}$ DM produced by freeze-in. The observed relic abundance occurs in the unshaded region for values of $T_{\mathrm{RH}}^{\mathrm{inf}}$ shown by the dashed red contours. Constraints from small scale structure are shown in green, with projections from future probes of small scale structure using the $21 \mathrm{~cm}$ line in dashed green. In the blue region $N_{1}$ decays too rapidly via $W_{R}$ to $\ell^{ \pm} \pi^{\mp}$, and in the pink region $N_{1}$ decays too rapidly via $W_{R}-W_{L}$ mixing to $\nu \gamma$ when $\mathrm{SU}(2)_{L}$ is broken by $(2,1)+(1,2)$ (solid) or by $(2,2)$ (dashed). The decay via $W_{R}-W_{L}$ mixing to $\ell^{+} \ell^{-} \nu$ is weaker and not shown. The horizontal dashed blue lines show the limit (3.4) on the mixing angle of $N_{1}$ with active neutrinos. Collider searches for $W_{R}$ exclude $v_{R}$ below about $10 \mathrm{TeV}$, as shown in orange.

mixing, (3.7), overproduces the observed amount of galactic gamma-rays, respectively [30]. Similarly, the decay of $N_{1}$ via active-sterile mixing overproduces the observed galactic xrays and gamma-rays for the mixing angle $\sin ^{2} 2 \theta_{1}$ labeling the purple dotted contours. Unlike the $W_{R}$-mediated decay, which is fixed by $v_{R}$, the decay via $N_{1}-\nu$ mixing is set by the free parameter $\theta_{1}$. Lastly, searches at the LHC for heavy charged boson resonances $\left(p p \rightarrow W_{R} \rightarrow N_{1} \ell\right)[95]$ and neutral boson resonances $\left(p p \rightarrow Z_{R} \rightarrow \ell^{+} \ell^{-}\right)$[96] exclude $v_{R}$ below about $10 \mathrm{TeV}$, as shown by the orange region.

Figure 4 shows that the parameter space for $N_{1}$ DM from freeze-in is weakly constrained compared to that of $N_{1} \mathrm{DM}$ from freeze-out and dilution, shown in figure 1 . For example, $v_{R}$ could be as low as about $100 \mathrm{TeV}$, with the reheat temperature after inflation below $100 \mathrm{GeV}$. Likewise, bounds on $M_{1}$ are weak; although as $M_{1}$ increases $\sin ^{2} 2 \theta_{1}$ is constrained to become extremely small to keep $N_{1}$ sufficiently long-lived. However, if leptogenesis via $N_{2}$ decay is incorporated into the $N_{1}$ DM freeze-in cosmology, the $\left(M_{1}, v_{R}\right)$ parameter space becomes more tightly constrained. In a future work, we discuss this viable parameter space in the framework of Higgs Parity [72]. 


\section{Conclusions}

Since right-handed neutrinos $N_{i}$ have no SM gauge interactions, it is plausible that one of them, $N_{1}$, is sufficiently stable to make up dark matter. A theory containing $N_{i}$ has three types of neutrino masses: Dirac masses, $\left(\nu_{i} N_{j}\right)$, and Majorana masses, $\left(\nu_{i} \nu_{j}\right)$ and $\left(N_{i} N_{j}\right)$. In general, these are described by three independent mass matrices. In this paper we have studied theories with a LR symmetry that forces the Majorana mass matrices for $\left(\nu_{i} \nu_{j}\right)$ and $\left(N_{i} N_{j}\right)$ to be proportional. In simple theories with the $\mathrm{SU}(2)_{R}$ and $\mathrm{SU}(2)_{L}$ gauge groups broken by doublet vacuum expectation values of strength $v_{R}$ and $v$, the constant of proportionality is $v^{2} / v_{R}^{2}$, whereas in the conventional LR theory, with scalar triplets and bidoublets, the constant of proportionality is $c v^{2} / v_{R}^{2}$, with $c \lesssim 1$.

At sufficiently high temperatures in the early universe, $N_{i}$ are kept in thermal equilibrium via the $\mathrm{SU}(2)_{R}$ gauge interactions. The initially thermal $N_{1}$ can account for the observed DM if they are subsequently diluted by decays of the initially thermal $N_{2}$. We have shown that the $\left(v_{R}, M_{1}\right)$ parameter space for this simple origin of DM is highly restricted, and indeed bounded, as shown in figure 1. The allowed region is triangular with $v_{R} \simeq\left(10^{8}-3 \times 10^{12}\right) \mathrm{GeV}$ and $M_{1} \simeq(2 \mathrm{keV}-1 \mathrm{MeV})$ for $c=1$. As $c$ is reduced, the allowed region shrinks in size and shifts to lower values of $\left(v_{R}, M_{1}\right)$, disappearing entirely at $\left(10^{6} \mathrm{GeV}, 2 \mathrm{keV}\right)$ when $c \simeq 10^{-4}$.

Constraints that determine the lower bounds on $M_{1}$ and $v_{R}$ are straightforward, arising from requirements that DM not be too warm and $N_{2}$ decays without disturbing nucleosynthesis. However, there is a third constraint, which leads to upper bounds on both $M_{1}$ and $v_{R}$, and is involved. In appendix A we show that this DM scenario places constraints on the active neutrino masses in such a way that the mass of $N_{2}$ is determined by (4.9), and grows rapidly with $v_{R}$. Thus at large enough $v_{R}, N_{2}$ decays dominantly via $W_{R}$ exchange; the requirement that this decay is slow enough to sufficiently dilute $N_{1}$ places an upper bound on $M_{1} v_{R}$, as shown by the blue region of figure 1 .

Observational probes of this $N_{1} \mathrm{DM}$, from relativistic freeze-out and dilution by $N_{2}$ decay, are shown in figure 2 for $c=1$. The bulk of the $\left(v_{R}, M_{1}\right)$ parameter space is at lower values of $M_{1}$, leading to signals of warmness in large scale structure. Indeed, a significant portion of the parameter space can be observationally probed using $21 \mathrm{~cm}$ cosmology. A subdominant component of $N_{1} \mathrm{DM}$ is produced non-thermally via the $W_{R}$ beta decay $N_{2} \rightarrow N_{1} \ell^{+} \ell^{-}$, producing $N_{1}$ that become non-relativistic at temperatures $\mathcal{O}(\mathrm{eV})$ and are therefore hot. The size of this component is proportional to $\left(M_{1} v_{R}\right)^{2}$ and, coincidentally, present limits on this hot DM component are close to the previously described limit on $M_{1} v_{R}$ from too much $N_{1}$ DM. Indeed, the interesting case of $N_{2}$ decaying dominantly via $W_{R}$ is already in tension with observation, and future CMB measurements will thoroughly probe this possibility. During the era of $N_{2}$ matter domination, density perturbations on small enough scales grow and could potentially lead to observable structures. Unfortunately, for pulsar timing arrays to see a signal in the region of reheat temperatures above the $4 \mathrm{MeV}$ BBN bound, requires $M_{1}>\mathrm{MeV}$, which is excluded by insufficient dilution of $N_{1}$. 
Given that the decays of $N_{2}$ are out of thermal equilibrium, it is plausible that they lead to leptogenesis. We explore this is detail in a future publication [72], and here we simply observe that sufficient baryon asymmetry arises only if such decays are early enough, as shown by the dashed purple line in figure 2. A large fraction of the parameter space that allows leptogenesis can be probed by $21 \mathrm{~cm}$ cosmology.

The $\mathrm{SU}(2) \times \mathrm{SU}(2)_{R} \times \mathrm{U}(1)_{B-L}$ gauge group studied in this paper provides an elegant setting for Higgs Parity [12-15], which correlates the SM parameters including the top quark and Higgs boson masses and the QCD coupling constant with the scale of $\mathrm{SU}(2)_{R}$ breaking. The predicted top quark mass in this scheme is consistent with the experimentally preferred value of it for $v_{R}$ in the range of $\left(10^{9}-10^{13}\right) \mathrm{GeV}$, as shown in figure 3, which includes much of the range relevant for $N_{1}$ DM. As uncertainties in the Higgs mass and the QCD coupling are reduced in near future measurements, $v_{R}$ is predicted within a factor of 10 . It will be interesting to see whether the ranges of $v_{R}$ for Higgs Parity and $N_{1}$ DM remain consistent. Precise measurements of the top quark mass at future linear colliders such as ILC can predict $v_{R}$ with an accuracy of a few tens of percent. The range of $v_{R}$ that gives precision gauge coupling unification is also shown in figure 3; remarkably it is consistent with Higgs Parity and much of the range needed for $N_{1}$ DM. An important question is how easily the conditions for cosmological stability of $N_{1}$ can be implemented in a realistic $\mathrm{SO}(10)$ theory of flavor.

In LR theories, if the reheat temperature after inflation is too low for $W_{R}$ exchange to put $N_{i}$ into thermal equilibrium, the $N_{1}$ DM abundance can be successfully generated by freeze-in, as shown by the solid red contours in figure 4 . In this case the scale $v_{R}$ is unconstrained, except by direct limits from LHC on the masses of $W_{R}$ and $Z_{R}$. There are, however, strong limits on $M_{1}$ from warmness and from $N_{1}$ stability requirements.

\section{Acknowledgments}

We thank Bibhushan Shakya for useful discussion. This work was supported in part by the Director, Office of Science, Office of High Energy and Nuclear Physics, of the US Department of Energy under Contracts DE-AC02-05CH11231 (JD and LJH) and DESC0009988 (KH), as well as by the National Science Foundation under grants PHY-1316783 and PHY-1521446 (LJH).

\section{A Neutrino mass relations}

In this appendix, we show the constraints on the mass eigenvalues of the active neutrinos through the requirements of abundance and radiative stability of $N_{1}$ DM, together with cosmological bounds on the warmness of $N_{1}$ and the reheating temperature from $N_{2}$ decay. We remind the reader that we work in a mass basis for $N_{i}$, which have masses $M_{i}$. The states $\nu_{i}$ are related to $N_{i}$ by LR symmetry, and are not necessarily mass eigenstates.

We first consider the case $M_{3}>M_{1}$. Constraints on the Yukawa matrix $y_{i j}$, and lower bounds on $M_{1}$ and $M_{2}$, then ensure that the seesaw mechanism is operative, so that the $\nu_{i}$ 
mass matrix is

$$
m_{i j}=\delta_{i j} c \frac{v^{2}}{v_{R}^{2}} M_{i}-\sum_{k=1}^{3} \frac{y_{i k} y_{j k}}{M_{k}} v^{2}
$$

as in (2.5). We will demonstrate two claims:

Claim 1. The lightest eigenstate is aligned with $\nu_{1}$, with mass $m_{1} \ll \sqrt{\Delta m_{\text {sol }}^{2}} \simeq 0.01 \mathrm{eV}$.

Claim 2. The mass of $N_{2}$ is determined by $M_{2} \simeq \mu\left(v_{R} / v\right)^{2} c^{-1}$, where $0.01 \mathrm{eV} \lesssim \mu \lesssim$ $0.10 \mathrm{eV}$. This is key to constraining the parameter space of frozen-out $N_{1} \mathrm{DM}$.

The stability of $N_{1}$ and $N_{2}$ require $\left|y_{1 i}\right|,\left|y_{2 i}\right| \ll 1$, as indicated by eqs. (3.3) and (4.8), implying that the seesaw contributions from $N_{1}$ and $N_{2}$ exchange are both much less than $0.01 \mathrm{eV}$. Hence, to an excellent approximation, eq. (A.1) can be written as

$$
m_{i j} \simeq\left(\begin{array}{ccc}
c\left(\frac{v}{v_{R}}\right)^{2} M_{1}-\frac{y_{13}^{2}}{M_{3}} v^{2} & -\frac{y_{23} y_{13}}{M_{3}} v^{2} & -\frac{y_{13} y_{33}}{M_{3}} v^{2} \\
-\frac{y_{23} y_{13}}{M_{3}} & c\left(\frac{v}{v_{R}}\right)^{2} M_{2}-\frac{y_{23}^{2}}{M_{3}} v^{2} & -\frac{y_{23} y_{33}}{M_{3}} v^{2} \\
-\frac{y_{13} y_{33}}{M_{3}} v^{2} & -\frac{y_{23} y_{33}}{M_{3}} v^{2} & c\left(\frac{v}{v_{R}}\right)^{2} M_{3}-\frac{y_{33}^{2}}{M_{3}} v^{2}
\end{array}\right) .
$$

Next we find that the entry $m_{11}$ is much smaller than $\sqrt{\Delta m_{\mathrm{sol}}^{2}}$ :

$$
\begin{aligned}
c\left(\frac{v}{v_{R}}\right)^{2} M_{1} & \leq v^{2} M_{1}\left(\frac{1536 \pi^{3}}{14 M_{2}^{5} M_{\mathrm{Pl}}}\left(\frac{\pi^{2} g_{*}\left(T_{\mathrm{RH}}\right)}{10}\right)^{1 / 2}\left(\frac{\rho_{\mathrm{DM}} / s M_{2}}{1.6 \frac{3}{4} M_{1}}\right)^{2}\right)^{1 / 2} \quad\left(N_{2} \text { stability }\right) \\
& =6 \times 10^{-6} \mathrm{eV}\left(\frac{24 \mathrm{GeV}}{M_{2}}\right)^{3 / 2}\left(\frac{g_{*}\left(T_{\mathrm{RH}}\right)}{10.9}\right)^{1 / 4}, \\
\frac{\left|y_{13}\right|^{2}}{M_{3}} v^{2} & \leq \frac{M_{1}^{2}}{M_{3}} \sin ^{2} 2 \theta_{1} \\
& \leq 8 \times 10^{-5} \mathrm{eV}\left(\frac{2 \mathrm{keV}}{M_{1}}\right)^{4}\left(\frac{M_{1} / M_{3}}{1}\right) .
\end{aligned}
$$

Now we argue that $m_{13}$ is also negligible. The upper bound on $\left|y_{13}\right|$ of (3.3) from the stability of $N_{1}$ implies that $m_{13}$ is non-negligible only if $\left|y_{33}\right|$ is large, such that $\left|y_{33}\right|^{2} v^{2} / M_{3} \gg \sqrt{\Delta m_{\text {sol }}^{2}}$. To ensure that the observed sum of neutrino masses does not exceed $0.06-0.10 \mathrm{eV}, m_{33}$ must be tuned such that $\left|y_{33}\right|^{2} v^{2} / M_{3} \simeq c\left(v / v_{R}\right)^{2} M_{3}$. However,

$$
\begin{aligned}
\frac{\left|y_{13} y_{33}\right|}{M_{3}} v^{2} & \simeq \sqrt{c} \frac{\left|y_{31}\right| v^{2}}{v_{R}} \\
& \leq \sqrt{c} M_{1} \sin \theta_{1} \frac{v}{v_{R}} \\
& \leq M_{1} \sin \theta_{1} v\left(\frac{1536 \pi^{3}}{14 M_{2}^{5} M_{\mathrm{Pl}}}\left(\frac{\pi^{2} g_{*}\left(T_{\mathrm{RH}}\right)}{10}\right)^{1 / 2}\left(\frac{\rho_{\mathrm{DM}} / s M_{2}}{1.6 \frac{3}{4} M_{1}}\right)^{2}\right)^{1 / 4} \quad\left(N_{2} \text { stability }\right) \\
& \leq 2 \times 10^{-5} \mathrm{eV}\left(\frac{M_{1}}{2 \mathrm{keV}}\right)^{-2}\left(\frac{M_{2}}{24 \mathrm{GeV}}\right)^{-3 / 4}
\end{aligned}
$$

Hence, from the lower bounds on $M_{1,2}$ we conclude that $m_{13}$ is negligible. 
The mass matrix of the active neutrinos is therefore approximately

$$
m_{i j} \simeq\left(\begin{array}{ccc}
0 & -\frac{y_{23} y_{13}}{M_{3}} v^{2} & 0 \\
-\frac{y_{23} y_{13}}{M_{3}} & c\left(\frac{v}{v_{R}}\right)^{2} M_{2}-\frac{y_{23}^{2}}{M_{3}} v^{2} & -\frac{y_{23} y_{33}}{M_{3}} v^{2} \\
0 & -\frac{y_{23} y_{33}}{M_{3}} v^{2} & c\left(\frac{v}{v_{R}}\right)^{2} M_{3}-\frac{y_{33}^{2}}{M_{3}} v^{2}
\end{array}\right) .
$$

We put further constraints on the mass matrix by considering the two cases of $M_{3}$ : greater than or less than $M_{2}$.

Case 1: $\boldsymbol{M}_{\mathbf{3}}>\boldsymbol{M}_{\mathbf{2}}$. For this case, the entry $m_{12}$ is negligible. This is because the upper bound on $y_{32}$ is

$$
\begin{aligned}
\left|y_{23}\right|^{2} & \leq \frac{1}{\Gamma_{0} M_{\mathrm{Pl}}}\left(\frac{\pi^{2} g_{*}\left(T_{\mathrm{RH}}\right)}{10}\right)^{1 / 2}\left(\frac{M_{2} \rho_{\mathrm{DM}} / s}{1.6 \frac{3}{4} M_{1}}\right)^{2}, \\
\Gamma_{0} & \equiv \begin{cases}\frac{171 / 8}{1536 \pi^{3}} \frac{M_{2}^{3}}{v^{2}} & M_{2}<v \\
\frac{1}{8 \pi} M_{2} & M_{2}>v,\end{cases}
\end{aligned}
$$

so that

$$
\begin{aligned}
\left|m_{12}\right| & =\frac{\left|y_{13} y_{23}\right| v^{2}}{M_{3}} \\
& \leq \sin \theta_{1} v\left(\frac{1}{\Gamma_{0} M_{\mathrm{Pl}}}\left(\frac{\pi^{2} g_{*}\left(T_{\mathrm{RH}}\right)}{10}\right)^{1 / 2}\left(\frac{\rho_{\mathrm{DM}} / s}{1.6 \frac{3}{4}}\right)^{2}\right)^{1 / 2} .
\end{aligned}
$$

(Stability of $N_{1}$ and $N_{2}, M_{3}>M_{2}$ )

$$
\leq 9 \times 10^{-10} \mathrm{eV}\left(\frac{M_{1}}{2 \mathrm{keV}}\right)^{-5 / 2}\left(\frac{M_{2}}{24 \mathrm{GeV}}\right)^{-3 / 2}
$$

Next we show that $m_{23}$ is also small. The upper bound on $y_{33}$ is

$$
\begin{array}{rlr}
\left|y_{33}\right|^{2} & =\frac{M_{3}^{2}}{v^{2}}\left|\frac{m_{22}+\frac{y_{23}^{2}}{M_{3}} v^{2}}{M_{2}}-\frac{m_{33}}{M_{3}}\right| & \text { (Rewriting } \left.m_{33}\right) \\
& \leq \frac{M_{3}^{2}}{v^{2}}\left(\left|\frac{m_{22}}{M_{2}}\right|+\left|\frac{y_{23}^{2} v^{2}}{M_{2} M_{3}}\right|+\left|\frac{m_{33}}{M_{3}}\right|\right) & \text { (Triangle inequality) } \\
& \leq \frac{M_{3}^{2}}{v^{2} M_{2}}\left(\left|m_{22}\right|+\left|\frac{y_{23}^{2} v^{2}}{M_{2}}\right|+\left|m_{33}\right|\right) & \left(M_{2}<M_{3}\right) \\
& \lesssim \frac{M_{3}^{2} \sum m_{i}}{v^{2}} \frac{\text { (Upper bound on } m_{22}}{M_{2}} & \text { and } m_{33}, N_{2} \text { stability) }
\end{array}
$$


Hence, $m_{23}$ is at most

$$
\left|m_{23}\right| \leq v \sqrt{\left(\frac{1}{\Gamma_{0} M_{\mathrm{Pl}}}\left(\frac{\pi^{2} g_{*}\left(T_{\mathrm{RH}}\right)}{10}\right)^{1 / 2}\left(\frac{M_{2} \rho_{\mathrm{DM}} / s}{1.6 \frac{3}{4} M_{1}}\right)^{2}\right)\left(\frac{\sum m_{i}}{M_{2}}\right)} .
$$

Figure 5 (left) shows the region where the right-side of eq. (A.9) is greater than $\sqrt{\Delta m_{\text {sol }}^{2}}$ in the $M_{1}-M_{2}$ plane. As can be seen, everywhere in the cosmologically allowed region $\left|m_{23}\right| \ll \sqrt{\Delta m_{\text {sol }}^{2}}$. In the active neutrino mass matrix, only $m_{22}$ and $m_{33}$ can be comparable to the observed neutrino masses; for $M_{3}>M_{2}$ the $\nu_{i}$ basis is accurately the mass basis. The lightest active neutrino mass $m_{1}$ is much smaller than $\sqrt{\Delta m_{\mathrm{sol}}^{2}}$, showing Claim 1 .

The two heavier active neutrino masses $\left(m_{2}, m_{3}\right)$ are simply given by

$$
\begin{aligned}
& m_{2} \simeq m_{22}=c\left(\frac{v}{v_{R}}\right)^{2} M_{2}-\frac{y_{23}^{2}}{M_{3}} v^{2} \\
& m_{3} \simeq m_{33}=c\left(\frac{v}{v_{R}}\right)^{2} M_{3}-\frac{y_{33}^{2}}{M_{3}} v^{2}
\end{aligned}
$$

Furthermore,

$$
\begin{aligned}
\frac{\left|y_{23}\right|^{2}}{M_{3}} v^{2} & \leq \frac{\left|y_{23}\right|^{2}}{M_{2}} v^{2} \\
& \ll \sqrt{\Delta m_{\text {sol }}^{2}} .
\end{aligned}
$$

$\left(N_{2}\right.$ stability)

Therefore, we obtain Claim 2, with $\mu$ identified as $m_{2}$, the mass of $\nu_{2}$

$$
M_{2} \simeq m_{2}\left(\frac{v_{R}}{v}\right)^{2} \frac{1}{c} .
$$

Case 2: $\boldsymbol{M}_{\mathbf{3}}<\boldsymbol{M}_{\mathbf{2}}$. We first show that $\left|y_{23}\right|^{2} v^{2} / M_{3}$ cannot be larger than the active neutrino mass by contradiction. Let us assume that $\left|y_{23}\right|^{2} v^{2} / M_{3}$ is larger than the active neutrino mass. Then to suppress $m_{22}$, we need

$$
\frac{\left|y_{23}\right|^{2}}{M_{3}} v^{2} \simeq c\left(\frac{v}{v_{R}}\right)^{2} M_{2}
$$

If $\left|y_{33}\right|$ is larger than $\left|y_{23}\right|,\left|y_{33}\right|^{2} v^{2} / M_{3}$ is also larger than the active neutrino mass and must be cancelled by $c M_{3}\left(v / v_{R}\right)^{2}$, which is impossible since $M_{3}<M_{2}$. We conclude that $\left|y_{33}\right|<\left|y_{22}\right|$, which is used later.

Since $M_{3}>M_{1}$, the case where $N_{3}$ decays after matter-radiation equality is excluded due to entropy production by the decay, or too much $N_{3}$ DM if $N_{3}$ is cosmologically stable. We thus assume that $N_{3}$ decays before matter-radiation equality.

Case 2-1: $\boldsymbol{M}_{\mathbf{2}}<\boldsymbol{v}$. Since $\left|y_{33}\right|<\left|y_{23}\right|$ and $\left|y_{13}\right|$ is small, the decay of $N_{3}$ by $W_{L}$ exchange is determined by $y_{23}$. Then the decay rate of $N_{3}$ by $W_{R}$ exchange is negligible. In fact, if $N_{3}$ decays dominantly by $W_{R}$ exchange,

$$
\left|y_{23}\right|^{2} \frac{M_{3}^{3}}{v^{2}}<\frac{M_{3}^{5}}{v_{R}^{4}}
$$


In this case, however,

$$
\begin{aligned}
\frac{\left|y_{23}\right|^{2} v^{2}}{M_{3}} & \leq M_{2} v^{4}\left(\frac{1536 \pi^{3}}{14 M_{2}^{5} M_{\mathrm{Pl}}}\left(\frac{\pi^{2} g_{*}\left(T_{\mathrm{RH}}\right)}{10}\right)^{1 / 2}\left(\frac{\rho_{\mathrm{DM}} / s M_{2}}{1.6 \frac{3}{4} M_{1}}\right)^{2}\right)\left(N_{2} \text { stability, } M_{2}>M_{3}\right) \\
& =2 \times 10^{-7} \mathrm{eV}\left(\frac{M_{2}}{24 \mathrm{GeV}}\right)^{-2}\left(\frac{M_{1}}{2 \mathrm{keV}}\right)^{-2}
\end{aligned}
$$

which is in contradiction. Thus $N_{3}$ decays dominantly by $y_{32}$.

In order for $N_{2}$ to be the diluter (by definition), it must be that

$$
\begin{aligned}
& \frac{M_{2}}{\sqrt{\Gamma_{N_{2}}}}>\frac{M_{3}}{\sqrt{\Gamma_{N_{3}}}}, \\
& \frac{M_{2}}{\sqrt{\left|y_{23}\right|^{2} M_{2}^{3}}}>\frac{M_{3}}{\sqrt{\left|y_{23}\right|^{2} M_{3}^{3}}} \\
& \Rightarrow M_{3}>M_{2},
\end{aligned}
$$

which is a contradiction with our assumption that $M_{3}<M_{2}$.

Case 2-2: $\boldsymbol{M}_{\mathbf{2}}>\boldsymbol{v}$. When $M_{2}>v, N_{2}$ decays to $\ell H$ via $y_{2 i}$ or beta-decays via $W_{R}$ exchange. Both decay channels limit $\left|y_{23}\right|^{2} v^{2} / M_{3}$ to ensure $N_{2}$ is long-lived enough to provide dilution of $N_{1}$.

From the decay via $y_{2 i}$,

$$
\frac{\left|y_{23}\right|^{2}}{M_{3}} v^{2} \leq \frac{v^{2}}{M_{3}} \frac{8 \pi}{M_{2} M_{\mathrm{Pl}}}\left(\frac{\pi^{2} g_{*}\left(T_{\mathrm{RH}}\right)}{10}\right)^{1 / 2}\left(\frac{\rho_{\mathrm{DM}} / s M_{2}}{1.6 \frac{3}{4} M_{1}}\right)^{2}
$$

( $N_{2}$ stability)

To be compatible with our assumption that $\left|y_{23}\right|^{2} v^{2} / M_{3}>m_{1}+m_{2}+m_{3}$, it is required that

$$
\begin{aligned}
\frac{M_{3}}{M_{2}} & <\frac{8 \pi v^{2}}{M_{\mathrm{Pl}} \sum m_{i}}\left(\frac{\pi^{2} g_{*}\left(T_{\mathrm{RH}}\right)}{10}\right)^{1 / 2}\left(\frac{\rho_{\mathrm{DM}} / s}{1.6 \frac{3}{4} M_{1}}\right)^{2} \\
& =2 \times 10^{-9}\left(\frac{\sqrt{\Delta m_{\mathrm{atm}}^{2}}}{\sum m_{i}}\right)\left(\frac{g_{*}\left(T_{\mathrm{RH}}\right)}{106.5}\right)^{1 / 2}\left(\frac{2 \mathrm{keV}}{M_{1}}\right)^{2} .
\end{aligned}
$$

The turquoise shaded region in figure 5 (right) violates this condition for the minimum value of $M_{1}=2 \mathrm{keV}$; larger $M_{1}$ enlarges the region. From the decay via $W_{R}$ exchange,

$$
\begin{array}{rlrl}
\frac{\left|y_{23}\right|^{2}}{M_{3}} v^{2} & \leq M_{2}\left(\frac{v}{v_{R}}\right)^{2} & & \text { (Since } c \leq 1) \\
& \leq v^{2}\left(\frac{1536 \pi^{3}}{20 M_{2}^{3} M_{\mathrm{Pl}}}\left(\frac{\pi^{2} g_{*}\left(T_{\mathrm{RH}}\right)}{10}\right)^{1 / 2}\left(\frac{\rho_{\mathrm{DM}} / s M_{2}}{1.6 \frac{3}{4} M_{1}}\right)^{2}\right)^{1 / 2} \cdot \quad\left(N_{2} \text { stability }\right)
\end{array}
$$

In the purple region of figure 5 (right), the inequality is less than $\sum m_{i}$, also for the minimum value of $M_{1}=2 \mathrm{keV}$. 
There are additional constraints on $M_{2}$ and $M_{3}$ if $N_{3}$ decays after BBN. This occurs when

$$
\Gamma_{N_{3}} \simeq(2-20) \times \frac{1}{1536 \pi^{3}} \frac{M_{3}^{3}}{v^{2}}\left|y_{23}^{2}\right|<(0.1 \mathrm{sec})^{-1}
$$

where the coefficient depends on the kinematically available final states. If $M_{3}>$ few $\mathrm{MeV}$, then the decay products of $N_{3}$ carry enough energy to dissociate light elements formed during BBN, altering their relic abundances (see [97] and references therein). ${ }^{10}$ If $M_{3} \lesssim \mathrm{MeV}$, the decay after BBN does not necessarily dissociate any light elements, but can still alter their relic abundance if $N_{3}$ is long-lived enough to induce a matter-dominated era before decaying. This occur when

$$
\Gamma_{N_{3}}<\left(\frac{\pi^{2} g_{*}}{10}\right)^{1 / 2} \frac{1}{M_{\mathrm{Pl}}}\left(\frac{M_{3}}{M_{1}} \frac{\rho_{\mathrm{DM}}}{s}\right)^{2} .
$$

These constraints are shown as the orange region of figure 5 (right), where we use the upper bound on $\left|y_{23}\right|$ from the stability of $N_{2}$ as discussed above and use the decay rate for $M_{3} \gtrsim 2 m_{e}$. For smaller $M_{3}$, the actual decay rate is smaller and the abundance becomes larger. In the red shaded region of figure 5 (right), $N_{3}$ decays after it dominates the universe for the maximum value of $M_{1}=M_{3}$; smaller $M_{1}$ enlarges the region. In the union of orange and red shaded regions, $N_{3}$ creates entropy after BBN, which is excluded since the baryon abundance at $\mathrm{BBN}$ and at $\mathrm{CMB}$ would differ. We see that no parameter region is then consistent with $\left|y_{23}\right|^{2} v^{2} / M_{3}$ being larger than the active neutrino mass, completing the proof.

Since $\left|y_{23}\right|^{2} v^{2} / M_{3}$ is at the most as large as the active neutrino mass, with the upper bound on $\left|y_{13}\right|$ from the stability of $N_{1}, m_{23}$ is much smaller than the active neutrino mass. The active neutrino mass is almost 2 by 2 , showing Claim 1.

The range of $c M_{2}\left(v / v_{R}\right)^{2}$ is constrained. It cannot be much larger than the active neutrino mass; since $\left|y_{23}\right|^{2} v^{2} / M_{3}$ is at the most as large as the active neutrino mass, $m_{22}$ cannot be fine-tuned to be small enough. If $c M_{2}\left(v / v_{R}\right)^{2}$ is much smaller than the active neutrino mass, $c M_{3}\left(v / v_{R}\right)^{2}$ is also small. Then the active neutrino mass matrix is dominantly given by the see-saw from $M_{3}$, and two active neutrinos remain massless, which is in contradiction with observations. The only possibility is that $c M_{2}\left(v / v_{R}\right)^{2}$ is comparable to the active neutrino mass, showing Claim 2.

We next consider the case with $M_{3}<M_{1}$. The term $c\left(v / v_{R}\right)^{2} M_{3}$ is much smaller than the observed neutrino masses. The active neutrino mass matrix is dominantly given by the term $c\left(v / v_{R}\right)^{2} M_{2}$ and the seesaw from $N_{3}$, or a Dirac mass term with $N_{3}$ if $M_{3} \ll$ $0.1 \mathrm{eV}$, and hence is essentially rank-2. The lightest active neutrino mass is much lighter than $0.01 \mathrm{eV}$. If $c\left(v / v_{R}\right)^{2} M_{2}$ is smaller than the observed active neutrino mass, the active neutrino mass matrix is essentially rank-1 and cannot explain the observed active neutrino mass. Thus, it is required that $c\left(v / v_{R}\right)^{2} M_{2} \gtrsim \mu$. It is possible that $c\left(v / v_{R}\right)^{2} M_{2} \gg \mu$ if it

\footnotetext{
${ }^{10}$ For $M_{3}<100 \mathrm{MeV}$ hadronic decays of $N_{3}$ are absent and the effect on BBN only comes from photodissociation which is effective for $T<0.01 \mathrm{MeV}$. We find that $N_{3}$ decays below $T=0.01 \mathrm{MeV}$ for $M_{3}<$ $100 \mathrm{MeV}$.
} 

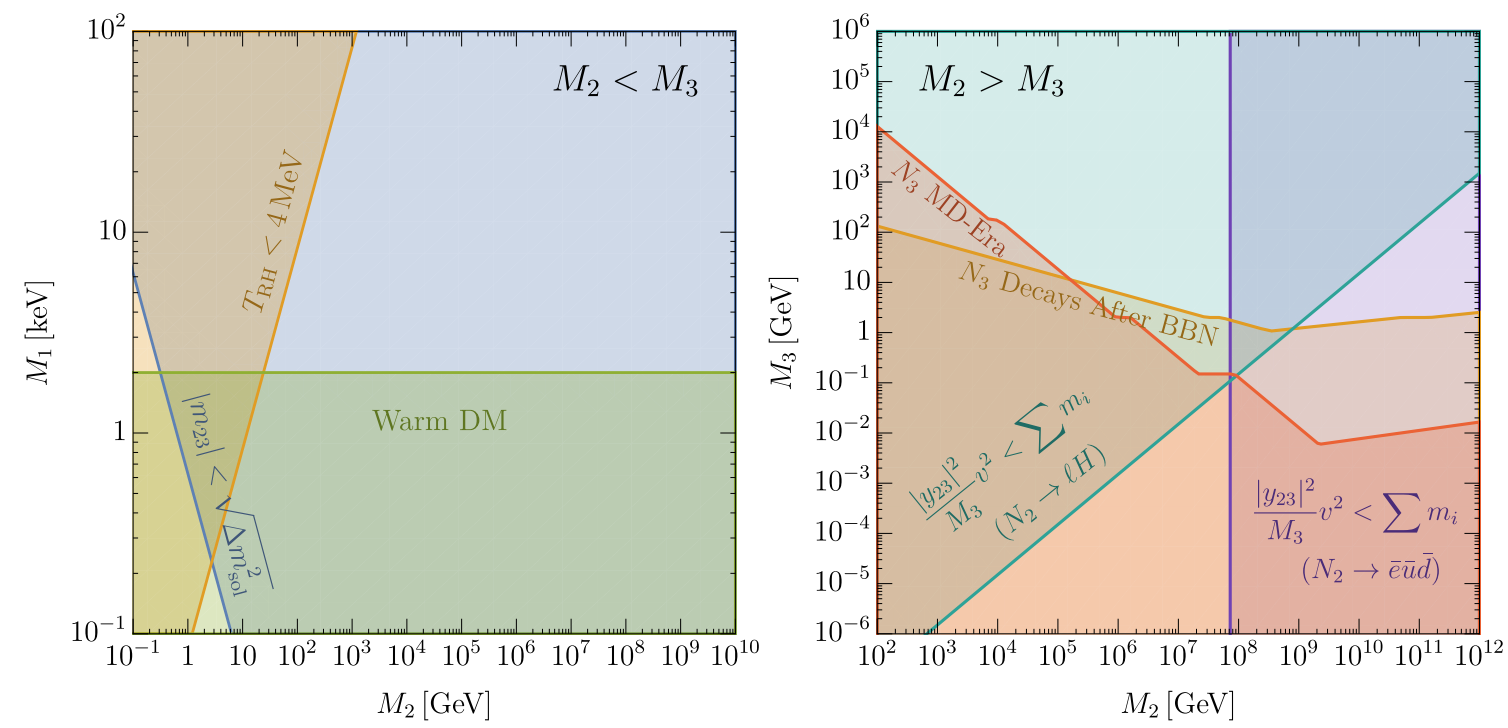

Figure 5. The right-handed neutrino mass parameter space showing the constraints which prove Claim 1 and Claim 2. Left: $M_{2}<M_{3}$ (Case 1) - the relation $M_{2}=m_{2}\left(v_{R} / v\right)^{2} / c$ is guaranteed if $\left|m_{23}\right|<\sqrt{\Delta m_{\text {sol }}^{2}}$. Stability of $N_{2}$ ensures $\left|m_{23}\right|<\sqrt{\Delta m_{\text {sol }}^{2}}$ in blue, which encompasses all of the parameter space not excluded by the warmness of DM (green) or Big Bang Nucleosynthesis (orange). Right: $M_{2}>M_{3}$ (Case 2) - the relation $M_{2}=\mu\left(v_{R} / v\right)^{2} / c$, where $0.01 \mathrm{eV} \lesssim \mu \lesssim$ $0.10 \mathrm{eV}$, is guaranteed if $\left|y_{23}\right|^{2} v^{2} / M_{3}<\sum m_{i}$. Stability of $N_{2}$ ensures $\left|y_{23}\right|^{2} v^{2} / M_{3}<\sum m_{i}$ in the purple and turqoise regions which encompass all of the parameter space not excluded from $N_{3}$ decaying after Big Bang Nucleosynthesis (orange). $N_{3}$ disrupts Big Bang Nucleosynthesis from the energy released in its decays when $M_{3}>1 \mathrm{MeV}$ in the orange region, and from the entropy produced from its decays in the intersection of the orange and red regions.

is cancelled by $\left|y_{23}\right|^{2} v^{2} / M_{3}$. The constraint on the case with $c\left(v / v_{R}\right)^{2} M_{2} \gg \mu$ is obtained by interpreting figure 1 with $c$ smaller than the actual value of $c$.

\section{B A symmetry for the cosmological stability of $N_{1}$}

For $N_{1}$ to make up dark matter, the mixing of active and sterile neutrinos must be very small to avoid limits on the radiative decay $N_{1} \rightarrow \nu \gamma$, as shown in (3.4). Sufficient stability can be a natural if a symmetry forbids the $\ell N_{1} H_{L}$ interaction in the effective theory (2.2), so that $y_{1 i}=0$. Any LR theory giving an effective theory below $v_{R}$ with no interactions for $N_{i}$ is particularly interesting: not only is $N_{1}$ cosmologically stable, but if $N_{2}$ has a mass significantly less than $v_{R}$, it is necessarily long-lived with a lifetime governed by $W_{R}$-mediated beta decay. In this case the allowed values of $v_{R}$ and $M_{1}$ are correlated - it is necessary to be on the blue line of figure 1 rather than in the unshaded triangle. Furthermore, since $N_{2}$ has a $10 \%$ branching ratio to decay to $N_{1}$, there is a component of DM that is hot, becoming non-relativistic around the eV era, with $\Delta N_{\text {eff }} \sim 0.1$ and $m_{\nu, \text { eff }} \sim 1.1 \mathrm{eV}$. As described in section 5.2, and shown in figure 2, this is close to present limits and will be discovered or refuted by CMB Stage IV [61]. 
For a LR model based on Higgs doublets $H_{L, R}$, such a symmetry must forbid the operator $\ell \bar{\ell} H_{L} H_{R}$, which leads to $\ell N H_{L}$, while allowing $\ell \bar{\ell} H_{L}^{\dagger} H_{R}^{\dagger}$, which yields the charged lepton Yukawa couplings $\ell \bar{e} H_{L}^{\dagger}$, as well as the Majorana mass operators $\ell \ell H_{L} H_{L}$ and $\bar{\ell} \bar{\ell} H_{R} H_{R}$. For example, this could be accomplished by a $Z_{4 L} \times Z_{4 R}$ symmetry with $\ell$ and $H_{L}$ transforming as $(i, 1)$ and $\left(\bar{\ell}, H_{R}\right)$ as $(1, i)$. The operator $q \bar{q} H_{L} H_{R}$ or $q \bar{q} H_{L}^{\dagger} H_{R}^{\dagger}$ is inconsistent with this $Z_{4 L} \times Z_{4 R}$ symmetry, so that the down and/or up-type quark Yukawa couplings must be generated by a different set of doublets, $H_{L, R}^{(q)}$, with the effective theory below $v_{R}$ containing the two doublets $H_{L}$ and $H_{L}^{(q)}$. A weak-scale Nambu-Goldstone boson is avoided by introducing a soft breaking of the $Z_{4 L} \times Z_{4 R}$ symmetry via the mass operator $H_{L}^{\dagger} H_{L}^{(q)}$.

Open Access. This article is distributed under the terms of the Creative Commons Attribution License (CC-BY 4.0), which permits any use, distribution and reproduction in any medium, provided the original author(s) and source are credited.

\section{References}

[1] J.C. Pati and A. Salam, Lepton Number as the Fourth Color, Phys. Rev. D 10 (1974) 275 [Erratum ibid. 11 (1975) ] [INSPIRE].

[2] R.N. Mohapatra and J.C. Pati, A Natural Left-Right Symmetry, Phys. Rev. D 11 (1975) 2558 [INSPIRE].

[3] G. Senjanović and R.N. Mohapatra, Exact Left-Right Symmetry and Spontaneous Violation of Parity, Phys. Rev. D 12 (1975) 1502 [InSPIRE].

[4] H. Georgi, The State of the Art-Gauge Theories, AIP Conf. Proc. 23 (1975) 575 [INSPIRE].

[5] H. Fritzsch and P. Minkowski, Unified Interactions of Leptons and Hadrons, Annals Phys. 93 (1975) 193 [INSPIRE].

[6] H. Georgi and D.V. Nanopoulos, Masses and Mixing in Unified Theories, Nucl. Phys. B 159 (1979) 16 [INSPIRE].

[7] T.W.B. Kibble, G. Lazarides and Q. Shafi, Walls Bounded by Strings, Phys. Rev. D 26 (1982) 435 [INSPIRE].

[8] M.A.B. Beg and H.-S. Tsao, Strong P, T Noninvariances in a Superweak Theory, Phys. Rev. Lett. 41 (1978) 278 [INSPIRE].

[9] R.N. Mohapatra and G. Senjanović, Natural Suppression of Strong $p$ and $t$ Noninvariance, Phys. Lett. B 79 (1978) 283 [inSPIRE].

[10] K.S. Babu and R.N. Mohapatra, CP Violation in Seesaw Models of Quark Masses, Phys. Rev. Lett. 62 (1989) 1079 [INSPIRE].

[11] K.S. Babu and R.N. Mohapatra, A Solution to the Strong CP Problem Without an Axion, Phys. Rev. D 41 (1990) 1286 [inSPIRE].

[12] L.J. Hall and K. Harigaya, Implications of Higgs Discovery for the Strong CP Problem and Unification, JHEP 10 (2018) 130 [arXiv:1803.08119] [INSPIRE].

[13] D. Dunsky, L.J. Hall and K. Harigaya, Higgs Parity, Strong CP and Dark Matter, JHEP 07 (2019) 016 [arXiv: 1902.07726] [INSPIRE]. 
[14] L.J. Hall and K. Harigaya, Higgs Parity Grand Unification, JHEP 11 (2019) 033 [arXiv: 1905.12722] [INSPIRE].

[15] D. Dunsky, L.J. Hall and K. Harigaya, Dark Matter, Dark Radiation and Gravitational Waves from Mirror Higgs Parity, JHEP 02 (2020) 078 [arXiv: 1908.02756] [INSPIRE].

[16] S. Dodelson and L.M. Widrow, Sterile-neutrinos as dark matter, Phys. Rev. Lett. 72 (1994) 17 [hep-ph/9303287] [inSPIRE].

[17] K. Perez, K.C.Y. Ng, J.F. Beacom, C. Hersh, S. Horiuchi and R. Krivonos, Almost closing the $\nu$ MSM sterile neutrino dark matter window with NuSTAR, Phys. Rev. D 95 (2017) 123002 [arXiv: 1609.00667] [INSPIRE].

[18] X.-D. Shi and G.M. Fuller, A New dark matter candidate: Nonthermal sterile neutrinos, Phys. Rev. Lett. 82 (1999) 2832 [astro-ph/9810076] [INSPIRE].

[19] F. Bezrukov, H. Hettmansperger and M. Lindner, keV sterile neutrino Dark Matter in gauge extensions of the Standard Model, Phys. Rev. D 81 (2010) 085032 [arXiv:0912.4415] [INSPIRE].

[20] S. Weinberg, Baryon and Lepton Nonconserving Processes, Phys. Rev. Lett. 43 (1979) 1566 [INSPIRE].

[21] T. Yanagida, Horizontal gauge symmetry and masses of neutrinos, [INSPIRE].

[22] M. Gell-Mann, P. Ramond and R. Slansky, Complex Spinors and Unified Theories, arXiv:1306.4669 [INSPIRE].

[23] P. Minkowski, $\mu \rightarrow e \gamma$ at a Rate of One Out of $10^{9}$ Muon Decays?, Phys. Lett. B 67 (1977) 421 [INSPIRE].

[24] R.N. Mohapatra and G. Senjanović, Neutrino Mass and Spontaneous Parity Nonconservation, Phys. Rev. Lett. 44 (1980) 912 [INSPIRE].

[25] M. Drewes et al., A White Paper on keV Sterile Neutrino Dark Matter, JCAP 01 (2017) 025 [arXiv: 1602.04816] [INSPIRE].

[26] K. Nandra et al., The Hot and Energetic Universe: A White Paper presenting the science theme motivating the Athena+ mission, arXiv:1306.2307 [INSPIRE].

[27] V. Tatischeff et al., The e-ASTROGAM gamma-ray space mission, Proc. SPIE Int. Soc. Opt. Eng. 9905 (2016) 99052N [arXiv: 1608.03739] [INSPIRE].

[28] A. Caputo, M. Regis and M. Taoso, Searching for Sterile Neutrino with X-ray Intensity Mapping, JCAP 03 (2020) 001 [arXiv: 1911.09120] [INSPIRE].

[29] E. Ma, Verifiable radiative seesaw mechanism of neutrino mass and dark matter, Phys. Rev. D 73 (2006) 077301 [hep-ph/0601225] [INSPIRE].

[30] R. Essig, E. Kuflik, S.D. McDermott, T. Volansky and K.M. Zurek, Constraining Light Dark Matter with Diffuse X-Ray and Gamma-Ray Observations, JHEP 11 (2013) 193 [arXiv: 1309.4091] [INSPIRE].

[31] L. Lavoura, General formulae for $f_{1} \rightarrow f_{2} \gamma$, Eur. Phys. J. C 29 (2003) 191 [hep-ph/0302221] [INSPIRE].

[32] A. Greljo, D.J. Robinson, B. Shakya and J. Zupan, $R\left(D^{(*)}\right)$ from $W^{\top}$ and right-handed neutrinos, JHEP 09 (2018) 169 [arXiv: 1804.04642] [INSPIRE]. 
[33] S. Tremaine and J.E. Gunn, Dynamical Role of Light Neutral Leptons in Cosmology, Phys. Rev. Lett. 42 (1979) 407 [INSPIRE].

[34] A. Boyarsky, O. Ruchayskiy and D. Iakubovskyi, A Lower bound on the mass of Dark Matter particles, JCAP 03 (2009) 005 [arXiv:0808.3902] [INSPIRE].

[35] D. Gorbunov, A. Khmelnitsky and V. Rubakov, Constraining sterile neutrino dark matter by phase-space density observations, JCAP 10 (2008) 041 [arXiv:0808.3910] [INSPIRE].

[36] V.K. Narayanan, D.N. Spergel, R. Dave and C.-P. Ma, Constraints on the mass of warm dark matter particles and the shape of the linear power spectrum from the Lya forest, Astrophys. J. Lett. 543 (2000) L103 [astro-ph/0005095] [INSPIRE].

[37] V. Iršič et al., New Constraints on the free-streaming of warm dark matter from intermediate and small scale Lyman- $\alpha$ forest data, Phys. Rev. D 96 (2017) 023522 [arXiv:1702.01764] [INSPIRE].

[38] C. Yèche, N. Palanque-Delabrouille, J. Baur and H. du Mas des Bourboux, Constraints on neutrino masses from Lyman-alpha forest power spectrum with BOSS and XQ-100, JCAP 06 (2017) 047 [arXiv: 1702.03314] [INSPIRE].

[39] U. Seljak, A. Makarov, P. McDonald and H. Trac, Can sterile neutrinos be the dark matter?, Phys. Rev. Lett. 97 (2006) 191303 [astro-ph/0602430] [INSPIRE].

[40] T. Asaka, M. Shaposhnikov and A. Kusenko, Opening a new window for warm dark matter, Phys. Lett. B 638 (2006) 401 [hep-ph/0602150] [INSPIRE].

[41] K. Harigaya and M. Kawasaki, QCD axion dark matter from long-lived domain walls during matter domination, Phys. Lett. B $\mathbf{7 8 2}$ (2018) 1 [arXiv:1802.00579] [INSPIRE].

[42] M. Kawasaki, K. Kohri and N. Sugiyama, Cosmological constraints on late time entropy production, Phys. Rev. Lett. 82 (1999) 4168 [astro-ph/9811437] [INSPIRE].

[43] M. Kawasaki, K. Kohri and N. Sugiyama, MeV scale reheating temperature and thermalization of neutrino background, Phys. Rev. D 62 (2000) 023506 [astro-ph/0002127] [INSPIRE].

[44] T. Hasegawa, N. Hiroshima, K. Kohri, R.S.L. Hansen, T. Tram and S. Hannestad, MeV-scale reheating temperature and thermalization of oscillating neutrinos by radiative and hadronic decays of massive particles, JCAP 12 (2019) 012 [arXiv: 1908.10189] [INSPIRE].

[45] K. Ichikawa, M. Kawasaki and F. Takahashi, The Oscillation effects on thermalization of the neutrinos in the Universe with low reheating temperature, Phys. Rev. D 72 (2005) 043522 [astro-ph/0505395] [INSPIRE].

[46] P.F. de Salas, M. Lattanzi, G. Mangano, G. Miele, S. Pastor and O. Pisanti, Bounds on very low reheating scenarios after Planck, Phys. Rev. D 92 (2015) 123534 [arXiv:1511.00672] [INSPIRE].

[47] M. Nemevšek, G. Senjanović and Y. Zhang, Warm Dark Matter in Low Scale Left-Right Theory, JCAP 07 (2012) 006 [arXiv: 1205.0844] [INSPIRE].

[48] D. Gorbunov and M. Shaposhnikov, How to find neutral leptons of the $\nu M S M$ ?, JHEP 10 (2007) 015 [Erratum ibid. 11 (2013) 101] [arXiv:0705.1729] [INSPIRE].

[49] J.R. Bond, G. Efstathiou and J. Silk, Massive Neutrinos and the Large Scale Structure of the Universe, Phys. Rev. Lett. 45 (1980) 1980 [INSPIRE]. 
[50] H. Sato and F. Takahara, Clustering of the Relic Neutrinos in the Expanding Universe, Prog. Theor. Phys. 64 (1980) 2029.

[51] F.R. Klinkhamer and C.A. Norman, Massive Neutrinos and Galaxy Formation, Astrophys. J. Lett. 243 (1981) L1 [INSPIRE].

[52] I. Wasserman, On the Linear Theory of Density Perturbations in a Neutrino Baryon Universe, Astrophys. J. 248 (1981) 1 [INSPIRE].

[53] K.A. Olive and M.S. Turner, Cosmological Bounds on the Masses of Stable, Right-handed Neutrinos, Phys. Rev. D 25 (1982) 213 [INSPIRE].

[54] SDSS collaboration, The Lyman-alpha forest power spectrum from the Sloan Digital Sky Survey, Astrophys. J. Suppl. 163 (2006) 80 [astro-ph/0405013] [INSPIRE].

[55] M. Viel, G.D. Becker, J.S. Bolton and M.G. Haehnelt, Warm dark matter as a solution to the small scale crisis: New constraints from high redshift Lyman- $\alpha$ forest data, Phys. Rev. D 88 (2013) 043502 [arXiv: 1306.2314] [INSPIRE].

[56] Planck collaboration, Planck 2018 results. VI. Cosmological parameters, arXiv: 1807.06209 [INSPIRE].

[57] N. Banik, G. Bertone, J. Bovy and N. Bozorgnia, Probing the nature of dark matter particles with stellar streams, JCAP 07 (2018) 061 [arXiv:1804.04384] [INSPIRE].

[58] J.B. Muñoz, C. Dvorkin and F.-Y. Cyr-Racine, Probing the Small-Scale Matter Power Spectrum with Large-Scale 21-cm Data, Phys. Rev. D 101 (2020) 063526 [arXiv: 1911.11144] [INSPIRE].

[59] L. Feng, J.-F. Zhang and X. Zhang, A search for sterile neutrinos with the latest cosmological observations, Eur. Phys. J. C 77 (2017) 418 [arXiv: 1703.04884] [INSPIRE].

[60] K. Abazajian et al., CMB-S4 Science Case, Reference Design and Project Plan, arXiv: 1907.04473 [INSPIRE].

[61] CMB-S4 collaboration, CMB-S4 Science Book, First Edition, arXiv:1610.02743 [INSPIRE].

[62] S. Böser et al., Status of Light Sterile Neutrino Searches, Prog. Part. Nucl. Phys. 111 (2020) 103736 [arXiv: 1906.01739].

[63] CORE collaboration, Exploring cosmic origins with CORE: Cosmological parameters, JCAP 04 (2018) 017 [arXiv: 1612.00021] [INSPIRE].

[64] A.L. Erickcek and K. Sigurdson, Reheating Effects in the Matter Power Spectrum and Implications for Substructure, Phys. Rev. D 84 (2011) 083503 [arXiv:1106.0536] [INSPIRE].

[65] K.-Y. Choi and T. Takahashi, New bound on low reheating temperature for dark matter in models with early matter domination, Phys. Rev. D 96 (2017) 041301 [arXiv:1705.01200] [INSPIRE].

[66] E.F. Keane et al., A Cosmic Census of Radio Pulsars with the SKA, PoS (AASKA14) 040 (2015) [arXiv: 1501.00056] [INSPIRE].

[67] J.A. Dror, H. Ramani, T. Trickle and K.M. Zurek, Pulsar Timing Probes of Primordial Black Holes and Subhalos, Phys. Rev. D 100 (2019) 023003 [arXiv:1901.04490] [INSPIRE].

[68] D. Croon, D. McKeen and N. Raj, Gravitational microlensing by dark matter in extended structures, Phys. Rev. D 101 (2020) 083013 [arXiv:2002.08962] [INSPIRE]. 
[69] Y. Bai, A.J. Long and S. Lu, Tests of Dark MACHOs: Lensing, Accretion and Glow, arXiv:2003.13182 [INSPIRE].

[70] E.W. Kolb and M.S. Turner, The Early Universe, Front. Phys. 69 (1990) 1.

[71] M. Fukugita and T. Yanagida, Baryogenesis Without Grand Unification, Phys. Lett. B 174 (1986) 45 [INSPIRE].

[72] D. Dunsky, L.J. Hall and K. Harigaya, Sterile Neutrino Dark Matter and Leptogenesis in Left-Right Higgs Parity, work in progress.

[73] R.T. Co, F. D'Eramo, L.J. Hall and D. Pappadopulo, Freeze-In Dark Matter with Displaced Signatures at Colliders, JCAP 12 (2015) 024 [arXiv: 1506.07532] [INSPIRE].

[74] J.A. Harvey and M.S. Turner, Cosmological baryon and lepton number in the presence of electroweak fermion number violation, Phys. Rev. D 42 (1990) 3344 [INSPIRE].

[75] D. Buttazzo et al., Investigating the near-criticality of the Higgs boson, JHEP 12 (2013) 089 [arXiv: 1307.3536] [INSPIRE].

[76] Particle Data Group collaboration, Review of Particle Physics, Phys. Rev. D 98 (2018) 030001 [INSPIRE].

[77] M. Cepeda et al., Report from Working Group 2, CERN Yellow Rep. Monogr. 7 (2019) 221 [arXiv: 1902.00134] [INSPIRE].

[78] G.P. Lepage, P.B. Mackenzie and M.E. Peskin, Expected Precision of Higgs Boson Partial Widths within the Standard Model, arXiv:1404.0319 [INSPIRE].

[79] TLeP Design Study Working Group collaboration, First Look at the Physics Case of TLEP, arXiv:1308.6176 [INSPIRE].

[80] K. Seidel, F. Simon, M. Tesar and S. Poss, Top quark mass measurements at and above threshold at CLIC, Eur. Phys. J. C 73 (2013) 2530 [arXiv: 1303.3758] [INSPIRE].

[81] T. Horiguchi et al., Study of top quark pair production near threshold at the ILC, arXiv: 1310.0563 [INSPIRE].

[82] Y. Kiyo, G. Mishima and Y. Sumino, Strong IR Cancellation in Heavy Quarkonium and Precise Top Mass Determination, JHEP 11 (2015) 084 [arXiv:1506.06542] [INSPIRE].

[83] M. Beneke, Y. Kiyo, P. Marquard, A. Penin, J. Piclum and M. Steinhauser, Next-to-Next-to-Next-to-Leading Order QCD Prediction for the Top Antitop S-Wave Pair Production Cross Section Near Threshold in $e^{+} e^{-}$Annihilation, Phys. Rev. Lett. 115 (2015) 192001 [arXiv: 1506.06864] [INSPIRE].

[84] The International Linear Collider Technical Design Report - Volume 2: Physics, arXiv:1306.6352 [INSPIRE].

[85] T.G. Rizzo and G. Senjanović, Grand Unification and Parity Restoration at Low-energies. 2. Unification Constraints, Phys. Rev. D 25 (1982) 235 [InSPIRE].

[86] F. Siringo, Grand unification in the minimal left-right symmetric extension of the standard model, Phys. Part. Nucl. Lett. 10 (2013) 94 [arXiv:1208.3599] [INSPIRE].

[87] Y. Kawamura, Gauge symmetry breaking from extra space $S^{1} / Z_{2}$, Prog. Theor. Phys. 103 (2000) 613 [hep-ph/9902423] [INSPIRE].

[88] Y. Kawamura, Triplet doublet splitting, proton stability and extra dimension, Prog. Theor. Phys. 105 (2001) 999 [hep-ph/0012125] [INSPIRE]. 
[89] G. Altarelli and F. Feruglio, SU(5) grand unification in extra dimensions and proton decay, Phys. Lett. B 511 (2001) 257 [hep-ph/0102301] [INSPIRE].

[90] L.J. Hall and Y. Nomura, Gauge unification in higher dimensions, Phys. Rev. D 64 (2001) 055003 [hep-ph/0103125] [INSPIRE].

[91] A. Hebecker and J. March-Russell, A Minimal $S^{1} /\left(Z_{2} \times Z_{2}^{\prime}\right)$ orbifold GUT, Nucl. Phys. B 613 (2001) 3 [hep-ph/0106166] [INSPIRE].

[92] L.J. Hall, Y. Nomura, T. Okui and D. Tucker-Smith, $\mathrm{SO}(10)$ unified theories in six-dimensions, Phys. Rev. D 65 (2002) 035008 [hep-ph/0108071] [INSPIRE].

[93] S. Biermann, A. Mütter, E. Parr, M. Ratz and P.K.S. Vaudrevange, Discrete remnants of orbifolding, Phys. Rev. D 100 (2019) 066030 [arXiv: 1906.10276] [INSPIRE].

[94] J. Heeck and D. Teresi, Cold keV dark matter from decays and scatterings, Phys. Rev. D 96 (2017) 035018 [arXiv: 1706. 09909] [INSPIRE].

[95] ATLAS collaboration, Search for a heavy charged boson in events with a charged lepton and missing transverse momentum from pp collisions at $\sqrt{s}=13 \mathrm{TeV}$ with the ATLAS detector, Phys. Rev. D 100 (2019) 052013 [arXiv:1906. 05609] [INSPIRE].

[96] ATLAS collaboration, Search for high-mass dilepton resonances using $139 \mathrm{fb}^{-1}$ of $\mathrm{pp}$ collision data collected at $\sqrt{s}=13 \mathrm{TeV}$ with the ATLAS detector, Phys. Lett. B 796 (2019) 68 [arXiv: 1903.06248] [INSPIRE].

[97] M. Kawasaki, K. Kohri and T. Moroi, Big-Bang nucleosynthesis and hadronic decay of long-lived massive particles, Phys. Rev. D 71 (2005) 083502 [astro-ph/0408426] [INSPIRE]. 